\title{
Supplementary Information for \\ The Mechanics of the Microtubule Seam \\ Interface Probed by Molecular Simulations and In Vitro Severing Experiments
}

Lukasz Szatkowski, ${ }^{\dagger}$ Dale R. Merz Jr., ${ }^{\dagger}$ Nan Jiang, ${ }^{\dagger}$ Ifunanya Ejikeme,${ }^{\dagger}$ Liudmila

Belonogov, ${ }^{\ddagger}$ Jennifer L. Ross, ${ }^{\ddagger}$ and Ruxandra I. Dima* ${ }^{* \dagger}$

${ }^{\dagger}$ Department of Chemistry, University of Cincinnati, Cincinnati, OH 45221

${ }^{\ddagger}$ Department of Physics, University of Massachusetts, Amherst, MA 01003

E-mail: dimari@ucmail.uc.edu 


\section{Supplemental Methods}

\section{I.1 Polymer Length and Finite Size Effects}

In vivo, microtubules (MTs) can be as long as tens of microns, and characterized by persistence lengths in. ${ }^{1}$ In atomistic-level simulations the maximal length that can be reached is 0.1 microns. To quantify the influence of finite size effects of the MTs on the results, we ran simulations on increasingly longer MTs, but limited by the computational expense. In order to push MTs in the middle, we clamped the ends of the filament. As a result, dimers located in the two end rings are artificially strengthened and they could also help enhance other nearby dimers. Therefore, the end rings are likely to experience less deformation under compression and become artificially brittle. For example, in the seam-down 8D MT case ${ }^{2}$ we fixed the outermost rings of dimers at the ends of the filament. This resulted in constraints placed on their nearest neighbor rings, meaning that only the four inner rings were truly free of outside imposed constraints. When turning the same filament upside down, because of the spiral geometry of MTs in the 13_3 lattice, we end up with fewer free dimer rings (two rings) than in the seam down orientation. To compensate for the loss of free rings, we extended MTs from 8D to 12D, as a 12D seam-up lattice with ends fixed has the same number of free rings as a seam-down $8 \mathrm{D}$ lattice with ends fixed. We also investigated MT lattices with 16D (16 dimers per PF) to evaluate the limit of finite-size effects. The majority of our runs were on the 12D lattices.

\section{I.2 Data Analysis: Force Indentation Curves and Contacts Analysis}

From each trajectory, we acquired the force-indentation curve. We used these curves, in combination with the percent native contacts and the time series of the protofilament (PF) bending angles discussed next, to pinpoint the first and critical breaking forces. These two significant barriers quantify how hard it is for severing proteins to begin MT cutting and ensure that it goes to completion (i.e., becomes irreversible). The process can be highly reversible between the initial and final cuts, depending on conditions, as shown in our previous work. ${ }^{2}$ We typically followed the indentation up to $20 \mathrm{~nm}$ depth, with the first break usually occurring at $8 \mathrm{~nm}$ and the critical break at $16-18 \mathrm{~nm}$.

To quantify the extent of fracturing (whether an interface is broken or not) we evaluated the native contacts using a tolerance up to $13 \AA$, above which the attractive potential term (Lennard-Jones) in Eqn.(1) from the main text becomes negligible. ${ }^{2}$ We calculated the normalized percentile of native contacts at every saved frame. We categorized each contact as internal (intra-chain or segment), intra-dimer (at the $\alpha$ and $\beta$ tubulin interface in a given dimer), or an external contact (one formed between the $\alpha$ or $\beta$ tubulin monomers of the investigated dimer to a neighboring $\alpha$ or $\beta$ tubulin). We further broke down the external contacts into their north, south, east, or west interface (only west, south, and east for the a tubulin monomer; only west, north, and east for the $\beta$ tubulin monomer). To determine the fraction of native contacts for each dimer, $Q_{n}$, we divided the number of native contacts present in any given conformation during the simulations by its initial value in the starting MT structure. Contact counts were typically 40-100 for the longitudinal interfaces (north and south) and 40-80 for the lateral interfaces (east and west). The overall $Q_{n}$ was the summation of all the external contacts, normalized to 1 . When only a singular lateral interface (for example, the western interface) broke, the overall $Q_{n}$ typically decreased from 1.0 to $0.7-0.8$. In contrast, for a longitudinal break (as an example, 2 western interfaces and a southern interface), the overall $Q_{n}$ typically decreases to $0.4-0.5$. The corresponding lateral or longitudinal $Q_{n}$ 's decrease to 0 . 
We determined the two breaking points that characterize each trajectory based on the drops in the various $Q_{n}$ 's (lateral, longitudinal, or overall). Namely, we most often selected the first break as the time when a lateral $Q_{n}$ decreased to 0 . Then the critical break would correspond to the time when the next significant decrease occurs

in $Q_{n}$. Typically, this was a longitudinal $Q_{n}$ decreasing to 0 , alone or in conjunction with further lateral $Q_{n}$ decreases. These breaking points very often matched exactly identifiable features in the force indentation curves. For example, the first break is often seen as an inflection or short plateau in these curves, whereas the critical break is often the global maximum (see Fig.S4). The exception was in the case of the simulations with the plate when the critical breaking corresponds only to a local maximum because for such trajectories the collision between the cantilever and the plate yields a nearly asymptotically growing force, as noted in our previous studies ${ }^{3}$ as well as in AFM indentation experiments. ${ }^{4}$ Another quantity whose time evolution can be used to pinpoint the critical force position is the PF bending angle, discussed below. Namely, we found that the time step corresponding to the sharpest change in the largest bending angle is also the time for the critical break.

For the retraction runs, we used the $Q_{n}$ analysis to determine whether the structure of the filament recovered fully or not upon the removal of the outside force.

The Relative $Q_{n}$ used for the comparison between native and non-native contacts was calculated similarly to the standard $Q_{n}$. The only difference is that for non-native contacts at each frame of trajectory we count all atoms within $8 \AA$ between two neighboring dimers for lateral $(E$ and $W$ ) and longitudinal interfaces $(N$ and $S)$. The calculated Relative $Q_{n}$ for an interface at each frame is the total number of contacts (native or non-native) divided by the average value of the native contacts for that type of interface (i.e., for lateral: 61 per interface, for longitudinal: 69 per interface, and for the seam: 42 per interface) in the starting configuration.

\section{I.3 Protofilament Bending Angle}

We measured the PF bending angle as the maximum angle obtained by constructing the vectors from the lowest (y-axis) monomer as the vertex to the preceding and succeeding (longitudinally) monomers that yielded the greatest angle. Moreover, we determined the bending angle for all 13 PFs only up the critical breaking point (see example in Fig.S4G for indentation position 1). We collected the angle array of the PF with the largest bending angle up to the critical breakpoint from all trajectories corresponding to a given setup. For each trajectory, the information about kinking angles was collected from the frame when the PFs began bending and up to the frame at which first longitudinal break occurs. Then we built the histograms (with $1^{\circ}$ bins) and the CDFs of kinking angles from the collected data. Finally we ran the comparison of the theoretical and experimental (for control or high salt conditions) CDFs in the Origin Pro software using the Kolmogorov-Smirnov test (KS). For more details see sections below and Fig.S10 and Fig.S11.

\section{MTs with both ends fixed}

To compare our bending angles with the angles obtained from experiments we collected the largest bending angles for each PF. Consequently, we chose for the MT bending angle at each time step the largest of the 13 PFs angles at that frame. For each frame and each PF, we measured all the angles using the two vectors passing between the following 3 points depicted in Fig.S2: 1) the center of mass of the first dimer in the PF (Fig.S2 panel A, ring R1), 2) the center of mass of each consecutive monomer in the PF, and 3) the center of mass of the last dimer in the PF (Fig.S2, panel A, ring R12). For each frame and for each PF we retained only the largest angle value, which is the angle between the vectors intersecting either at the $\alpha$ (panel $B$ ) or at the $\beta$ 
(panel C) tubulin monomer inside the PF. Additionally for each PF we determined the sign of the angle by comparing it with the angle form the starting frame of the trajectory formed between the first monomer, the center of mass of the whole MT, and the last monomer, which we choose to be negative. Therefore the angle for a PF bending inside the MT will always be negative, while any PF bending outside of the MT will have a positive angle. For the histograms we used only the absolute values of the angles. Additionally for the 8D polymer as a reference we used dimers from the R1 and R8 rings. For the 12D polymer we used three different reference system: R1-R12, R2-R11 and R3-R10 (which in length is similar to 8D polymer but without the influence of the frozen ends from the $8 \mathrm{D}$ case).

\section{MTs with the plus end free}

For the simulations of MTs with the plus end free because we released the constraints on the plus end previously frozen dimers were now able to move. Therefore any measurements with our previously described method for any MTs longer than 8D led to a large underestimation of the bending angle. For the 8D system we did not observe this effect because on the minus side we always overestimate the measured angles (because of the fixed end) and underestimate angles on the side of the plus end. For longer MTs with 12D and 16D, the changing position (movement) of the dimers at the plus end led to an extension of one of the vectors (from the center of the system to the plus end) resulting in a big underestimation of the angle on the plus side of the MT. At the same time, the measurement of the angle on the side of the frozen end of the lattice did not experience any bias. This led us to make a slight change in the angles measurement protocol. For the $12 \mathrm{D}$ polymer with the plus free end we measured the angle between the following 3 points: 1) the center of mass of the frozen dimer (first dimer on the minus end) shifted toward minus end by $50 \AA, 2$ ) the center of mass of the frozen dimer, and 3 ) the center of mass of the next monomer in the PF. However because this measurement covers only half of the MT length, we multiplied the resulting angle by 2 . Then we followed the procedure described above for MTs with both ends fixed.

\section{I.4 Protofilament Elongation}

All fittings procedures were performed in OriginPro software. We used four different schemes of non-linear fitting of our kinetic data (average elongation vs. time) to BoxLucas plot $y=a\left(1-e^{-b x}\right)$ which is a representation of first order kinetics:

1. using orthogonal distance regression algorithm without frozen parameter $a$ (both parameter $a$ and $b$ were fitted).

2. using orthogonal distance regression algorithm with frozen parameter a (the average value from all a values from the previous scheme)

3. using Levenberg-Marquardt algorithm (an iterative procedure which combines the Gauss-Newton method and the steepest descent method) without frozen parameter $a$ (both parameter $a$ and $b$ were fitted).

4. using Levenberg-Marquardt algorithm without frozen parameter a (the average value from all a values from the previous scheme)

An example of fitting plots can be found on Fig.S13A.

For examples of fitting the elongation time vs the polymer length (see Fig.S13B) we used linear regression $y=a x$ as it is implemented in the OriginPro Software. 


\section{Supplemental Results}

\section{II.1 Indentation pathways}

For the detailed description of the breaking pathways we will use the following scheme: lateral break, for example between 1st and 2nd PF - P1/P2; longitudinal break on PF, for example 3, will be P3(R4) where the number after $\mathrm{R}$ indicate after which ring, starting the count from the minus end, is the break.

\section{Simulations for MTs with both ends fixed}

$M T 8 D, p 3$ (indentation on the seam) - The initial breaking event corresponds to the loss of lateral interactions at the seam (in some cases together with the rip of longitudinal interfaces from two neighboring PFs located closest to the seam, P12 and P13), followed by breaks of multiple longitudinal interfaces from PFs in the neighborhood of the seam (P12, P13, P1 or/and P2 and P3). The simulations without the plate result in the largest number of breaks at the various interfaces. Thus, our results show that the plate delays the loss of MT structure under radial stress applied on the seam.

MT 12D, p3 (indentation on the seam) - Similar to the simulations for the MT 8D system, the breaking starts with the lateral rip of the seam. For the AHM/LHM models the second breaking event corresponds to longitudinal interfaces in PFs in the vicinity of the seam and in rings located under the tip, P1(R5), then P2(R5), P13(R6) and P12(R6). For the Reg/Top56 models, the second breaking event is at longitudinal interfaces in the PFs closest to the seam, P13(R5 or R6) and P1(R5). We found that only for the Reg model with plate the lateral break at the seam occurred after the lateral break between P5 and P6, which is the weakest lateral interface and in these simulations it is located directly over the plate. Next breaks for the Reg model are at longitudinal interfaces in P13(R6), P1(R5), P2(R5) and P12(R6). Moreover, we observed breaks in P13 at interfaces located close to the frozen plus end of the MT. Similar to the simulations for the shorter MT 8D system, we found that the absence of a plate leads to the largest number of breaks at the various interfaces. Snapshots for this type of runs are in Fig.S9B.

$M T 8 D, p 1-5$ - For all trajectories with and without plate there is a lot of similarity between the behavior of the two types of indentation setups: pushing between P1/P2 (for p5) and, respectively, between P12/P13 (for p1). We first found the loss of lateral contacts at the seam and at other interfaces on the sides of the respective indentation position (for p1: P1/P2 and P10/P11; for p5: P2/P3 and P11/P12). This is followed by the unzipping of longitudinal interfaces in the group of three neighboring PFs closest to the respective indentation point (for p1: P13, P12, and P11; for p5: P1, P2, and P3). In all cases, the longitudinal breaks start in order from the seam outward. Further pushing caused a lot of brutal breaks (without any visible pattern) on other PFs. Also for all trajectories with the plate and for indentation on position $\mathrm{p} 1$, we observe an almost instant initial lateral break of the P5/P6 interface, which is located on the plate and right opposite to the p1 position.

MT 12D, p1-p5 - Unlike the MT 8D case, for the longer MT filament we found two indentation pathways: one characterized by substantial buckling events and the second by the flattening of the lattice. The first pathway is very similar to the pathway described above for the 8D MTs. One difference between the pathways is that the first pathway (buckling) resulted in a lower first breaking force for simulations with the plate. For both pathways and set-ups (with and without the plate), the first event corresponds to the break of lateral interfaces: the seam and other lateral interfaces close to the indentation point (the only exception are trajectories for position $\mathrm{p} 1$ AHM with the plate where we observe lateral break between P12/P13 rather than on the seam). Later events 
correspond to a mixture of longitudinal and lateral breaks. Importantly, for all buckling trajectories, the longitudinal breaks start in order from the seam outward in three neighboring PFs, recalling the behavior of MT 8D. We also found that, after the first break, in the buckling trajectories one side of the MT moves away from the tip, while in the flattening trajectories the two PFs which start under the tip continue to move down with the tip. Finally, for the flattening pathway there is no clear breaking pattern beyond the first cut, as it results in number of brutal longitudinal breaks in various PFs and rings. Fig.S8B shows the evolution of the native and non-native contacts at the seam, at the pushed P12/P13 interface, and at the P5/P6 interface for pushing on p1. We found a similar behavior in Fig.S8C for pushing on P5. We note that the seam is still the interface that gains the largest percent of non-native contacts (up to 50\%), even if it is no longer the point of indentation. Another common element between all these trajectories is that the largest gain of non-native contacts occurs once the seam starts to rip (i.e., to lose native contacts). All the non-native contacts formed at the seam are still in the A-lattice configuration. Conformations along a typical buckling trajectory for the 12D case are in Fig.S9A.

$M T 8 D, p 2-p 4$ - The pathways followed by these simulations are the same irrespective of the presence of the plate. For both indentation positions the first event is the lateral rip at the seam. Further events correspond to multiple longitudinal breaks and lateral cracks in the PFs surrounding the seam. For pushing on P13, i.e., on position 2, the first event is the lateral break of the seam. Next, we saw longitudinal breaks in P13(R3), $\mathrm{P} 12$ (R3) and P1(R2). For pushing on P1, i.e., on position 4, the first event is the lateral break of the seam. Next, we saw longitudinal breaks in $\mathrm{P} 1(\mathrm{R} 3), \mathrm{P} 2(\mathrm{R} 3)$ and $\mathrm{P} 13(\mathrm{R} 4)$ and further multiple longitudinal breaks in $\mathrm{P} 2$, $\mathrm{P} 4, \mathrm{P} 12$ and P13. Thus a characteristic of these trajectories, distinct from the behavior of other indentation points, is that the late longitudinal breaks occur in the two PFs which are nearest neighbors of the pushed PF.

MT 12D, p2-p4 - All trajectories follow a very uniform pattern, similar to the MT 8D case reviewed above. The first event in all but one setup is the lateral break of the seam. The exception is for the simulations of indentation on position 2 (push on P13) with the plate where the first event corresponds to longitudinal breaks in P13 (in rings R6 and R7), followed by the lateral break of the seam. The next step is the longitudinal break of interfaces in the pushed PF, i.e., P13(R6 and R7) for position 2 and P1(R5 or R6) for position 4. The final step corresponds to the longitudinal break of two PFs adjacent to the pushed PF and the seam: P12(R6 or R7) and $\mathrm{P} 1(\mathrm{R} 5$ or R3) for position 2, and P2(R5 or R6) and P13(R6 or R7) for position 4.

$M T 8 D$, on $P 3$ - Similar to the other indentation simulations reviewed before, we found an initial flattening of the MT under the tip (ovalization of the cross-section). However this time we found two different pathways. For the main pathway (3 out of 4 , or $75 \%$ of trajectories) there is no time/frame separation between the initial and critical breaking events because both lateral (P2/P3 and/or P3/P4) and longitudinal (P3(R3) or P3(R4)) breaks on protofilament P3 occur almost simultaneously. Furthermore, shortly afterwards we observed the release of the whole dimer on which we push directly, followed by the release of its nearest neighbor longitudinal dimer (within 10-15 frames from the first event). Moreover, the seam and the P5/P6 lateral interfaces also break immediately after the initial longitudinal crack on P3. Some very modest unzipping of the seam and the P5/P6 lateral interfaces starts 100 frames before the initial crack on P3. However because this action amounts to a loss of less than $5 \%$ of the initial number of contacts, we do not treat it as a separate true first breaking event as in the trajectories reviewed above. Interestingly, the longitudinal break on P3 happens much earlier than the similar cracking for position p1 or p5 for the MT 8D lattice simulations. The second pathway is similar to the pathways described before: the first breaking event corresponds to a lateral break (of the P2/P3 interface) and the critical breaking event, which occurs after $\sim 100$ frames from the first one, corresponds to a longitudinal break (in ring 4 of P3, or P3(R4)) together with the lateral break of the P3/P4 interface. 


\section{Simulations for MTs with the plus end free}

MT 8D, SEAM down, pushing on the P6/P7 interface (from our previous work in ${ }^{2}$ ) - In all trajectories we saw the formation of a lateral crack between P5 and P6 (which is the weakest lateral interface in the Reg MT lattice), accompanied by the strong ovalization of the free plus end. These events lead to the movement of P5 to the side of the indentation sphere, while P6 remains under the tip. These movements allow for the full propagation of the lateral crack between P5 and P6 to the free plus end of the MT, accompanied by the shift of P6 by about the length of a dimer towards the frozen (minus) end of the lattice. Next, another lateral breaks occurs between P9 and P10. Eventually, at the time when the tip crushed on the plate, we observed longitudinal breaks in P6 and P7.

MT 12D, SEAM up, pushing on the seam (the P1/P13 interface) - In all trajectories we observed the formation of a lateral crack between P1 and P13 (at the seam), which is accompanied by the strong ovalization of the free plus end. Moreover we also observe the loss of lateral contacts between P5 and P6 which are now located right above the plate. The crack on the seam propagates to the free end of the filament. However the separation of the monomers on the last ring (R12) at the free end was more modest than in the shorter (8D) lattice. Some longitudinal cracks develop close to the frozen minus end, likely due to the reduced mobility of the rings at this end. Snapshots of main states along this pathway are in Fig.S9C.

MT 12D, SEAM down, pushing away of the seam (the P6/P7 interface) - This is the identical set-up from our previous work $^{2}$. Here we carried out simulations for the MT 12D filaments. We found two breaking pathways: without ( $1 / 3$ of runs) and with the propagation of the lateral break (2/3 of runs) to the free plus end. Both pathways start with the same event: the relaxation of the MT filament and the ovalization of the MT under the tip and at the free plus end. In the trajectory without propagation of the lateral break, PFs P5, P6, and P7 track the motion of the tip and bend inside the MT. The first lateral break occurs between P5 and P6 and propagates by a length of 3 dimers until P6 starts to break longitudinally. The longitudinal break propagates to neighboring PFs in order from P6 eastward (P7, P8 and P9), accompanied by a lateral crack between P9 and P10. At late stages, we saw lateral breaks between P6/P7 and at the seam (P1/P13). In trajectories experiencing the full propagation of the lateral crack, after the break of the P5/P6 lateral interface, P5 moves to the side of the tip and no longer follows the trajectory of the tip. This event allows the lateral break between P5/P6 to propagate all the way to the free end of the filament. Breaks at longitudinal interfaces start much later, almost at the time when the tip crushes PFs on the plate. They are accompanied by the lateral crack at the P9/P10 interface, recalling the behavior of the MT 8D filament described above. Evolution of native and non-native contacts for this pathway is in Fig.S8F. In this set-up the seam gains a large percent of non-native contacts $(\sim 75 \%)$ early in the trajectory and maintains them for the longest duration of any of the reviewed pathways. We assign this behavior to the interaction between the seam and the plate, as this is the only set-up where the seam rests directly over the plate. Representative states along the major pathway presented in Fig.S9D illustrate the full propagation of the crack at the P5/P6 lateral interface to the free plus end of the lattice. In contrast, for the run without propagation of the lateral break (Fig.S9D) the final conformation (frame 550) shows a gap on the upper surface of the lattice and longitudinal breaks in 4 neighboring PFs leading to the 2 PFs located closest to the side of the filament to bend outwards.

MT 12D, SEAM down, pushing away of the seam, pushing on P6 and P7 - All trajectories follow a pathway similar to the one corresponding to the lack of propagation of the lateral break from the MT 12D pushing on the P6/P7 interface case discussed above (Fig.S9D). The only difference is that the propagation of the P5/P6 lateral crack occurs over a longer distance resulting in fewer longitudinal breaks. Example of conformations for the stages in this pathway are in Fig.S9E. 


\section{II.2 The recovery of the MT lattice from indentation}

MTs are capable of recovering fast if we retract the cantilever after earlier breaking stages. This behavior was observed in prior AFM experiments. ${ }^{4}$ Here we will discuss in detail this self-healing process.

We divided our retraction simulations into two groups based on the number of broken dimers in the initial state: 1) if the initial state had up to 10 broken dimers (a broken dimer means that at least $16 \%$ of its inter-dimer contacts are lost), it was equivalent to starting the retraction after the initial breaking but before the critical breaking; 2) if the initial state had more than 10 broken dimers, it was equivalent to starting the retraction from conformations corresponding to the critical breaking point. Retraction simulations of type 1) are the first entry for each type of trajectory listed in Table S3, usually corresponding to retraction started after less than 300 indentation frames.

As shown in Table S3, retractions of type 1) started after fewer indentation steps or, equivalently, from conformations with modest breaks (at most 10 broken dimers) had a very high probability of recovery: 90\% of the 17 retractions simulated without a plate recover. In our previous study, ${ }^{2}$ we found out that originally defect-free MTs starting from this type of indented conformations would always recover. In contrast, only $\sim 80 \%$ of the 8D MTs originally with defects would recover upon retraction from such indented states. This is additional evidence that the seam does not behave like a missing subunit(s) MT lattice defect, and is an unlikely target of severing proteins. The lattice is able to recover fast from initial breaks around the seam during the pauses in the action of severing proteins. For the retraction runs that started from indented conformations with more than 10 broken dimers, we found that the MTs are unlikely to recover as $68 \%$ of the 22 different retraction runs fail to reach the intact MT conformation, in accord with our previous findings for defect-free MTs. ${ }^{2}$ 


\section{Supplemental Tables}

\section{Table S1. Average Breaking Forces, models with both end fixed}

We collected the breaking forces from the indentation runs, plotted the distributions (see the histogram figure), and calculated the respective averages by dimer length, indentation position, with and without plate for the first and critical break. The 8D MT always had a larger first breaking force than the 12D MT. We did not see any significant influence of the plate to either the first or the critical breaking forces.

\begin{tabular}{|c|c|c|c|}
\hline Position & $\begin{array}{l}\text { Length, } \\
\text { plate: } \\
\text { Y - Yes } \\
\text { N - No } \\
\text { B - both }\end{array}$ & $\begin{array}{l}\text { First breaking, } \\
\text { Avg. Force (nN) } \\
\text { (Std. Dev.); } \\
\text { \# of trajectories }\end{array}$ & $\begin{array}{l}\text { Critical breaking, } \\
\text { Avg. Force (nN) } \\
\text { (Std. Dev.); } \\
\text { \# of trajectories }\end{array}$ \\
\hline \multirow[t]{6}{*}{$1-5$} & $8 D, Y$ & 0.4657 (0.0273); 30 & 0.7365 (0.0689); 30 \\
\hline & $8 \mathrm{D}, \mathrm{N}$ & 0.4625 (0.0277); 31 & 0.7303 (0.0786); 31 \\
\hline & $8 \mathrm{D}, \mathrm{B}$ & 0.4641 (0.0273); 61 & 0.7333 (0.0734); 61 \\
\hline & 12D, Y & 0.3677 (0.0479); 45 & $0.7184(0.1066) ; 37$ \\
\hline & $12 \mathrm{D}, \mathrm{N}$ & $0.3576(0.0321) ; 43$ & 0.7063 (0.0807); 35 \\
\hline & 12D, B & 0.3627 (0.0410); 88 & 0.7125 (0.0944); 72 \\
\hline \multirow[t]{6}{*}{1,5} & $8 D, Y$ & $0.4861(0.0273) ; 10$ & $0.6822(0.0382) ; 10$ \\
\hline & $8 \mathrm{D}, \mathrm{N}$ & $0.4629(0.0217) ; 13$ & 0.6901 (0.0687); 13 \\
\hline & $8 D, B$ & $0.4730(0.0265) ; 23$ & $0.6867(0.0564) ; 23$ \\
\hline & 12D, Y & 0.4094 (0.0505); 15 & $0.6383(0.0768) ; 13$ \\
\hline & $12 \mathrm{D}, \mathrm{N}$ & $0.3787(0.0257) ; 18$ & 0.6725 (0.0905); 16 \\
\hline & 12D, B & 0.3926 (0.0413); 33 & $0.6572(0.0850) ; 29$ \\
\hline \multirow[t]{6}{*}{2,4} & $8 \mathrm{D}, \mathrm{Y}$ & $0.4454(0.0111) ; 12$ & $0.7172(0.0274) ; 12$ \\
\hline & $8 \mathrm{D}, \mathrm{N}$ & $0.4657(0.0323) ; 11$ & 0.7094 (0.0303); 11 \\
\hline & $8 \mathrm{D}, \mathrm{B}$ & $0.4552(0.0253) ; 23$ & $0.7135(0.0284) ; 23$ \\
\hline & 12D, Y & 0.3417 (0.0329); 18 & 0.7066 (0.0487); 16 \\
\hline & $12 \mathrm{D}, \mathrm{N}$ & $0.3350(0.0282) ; 17$ & $0.7042(0.0382) ; 13$ \\
\hline & 12D, B & $0.3384(0.0304) ; 35$ & 0.7055 (0.0436); 29 \\
\hline \multirow[t]{6}{*}{3} & $8 \mathrm{D}, \mathrm{Y}$ & 0.4707 (0.0246); 8 & 0.8333 (0.0323); 8 \\
\hline & $8 \mathrm{D}, \mathrm{N}$ & $0.4566(0.0331) ; 7$ & 0.8379 (0.0456); 7 \\
\hline & $8 \mathrm{D}, \mathrm{B}$ & $0.4641(0.0288) ; 15$ & $0.8354(0.0377) ; 15$ \\
\hline & 12D, Y & $0.3545(0.0246) ; 12$ & 0.8722 (0.0628); 8 \\
\hline & $12 \mathrm{D}, \mathrm{N}$ & 0.3582 (0.0194); 8 & 0.8010 (0.0419); 6 \\
\hline & 12D, B & $0.3560(0.0222) ; 20$ & $0.8417(0.0643) ; 14$ \\
\hline Prot 3 & $8 D, Y$ & $0.3590(0.0088) ; 3^{*, * *}$ & $0.5188(0.0237) ; 3^{* *}$ \\
\hline
\end{tabular}

${ }^{*}$ forces at the point when the seam start unzipping

${ }^{* *}$ data for the main trajectory with only critical breaking event, for the remaining trajectory where we could distinguish FBF and CBF the forces are, respectively: 0.436 and $0.580 \mathrm{nN}$. 


\section{Table S2. Average Breaking Forces, models with plus end free}

We used the same approach as above to calculate the average First Breaking Force and the Critical Breaking Force. For this type of simulations we always included the plate.

\begin{tabular}{|l|c|c|}
\hline \multicolumn{1}{|c|}{ Model } & $\begin{array}{c}\text { First breaking, } \\
\text { Avg. Force }(\mathrm{nN}) \\
\text { (Std. Dev.); } \\
\text { \# of trajectories }\end{array}$ & $\begin{array}{c}\text { Critical breaking, } \\
\text { Avg. Force (nN) } \\
\text { (Std. Dev.); } \\
\text { \# of trajectories }\end{array}$ \\
\hline 8D, SEAM up, P1P13 & $0.4773(0.0275) ; 3$ & $0.6142(0.0168) ; 3$ \\
\hline 8D, SEAM down, P6P7 & $0.4822(0.0195) ; 4$ & $0.5854(0.0297) ; 4$ \\
\hline 12D, SEAM up, P1P13* & $0.4777(0.0144) ; 4$ & $0.8103(0.0160) ; 4$ \\
\hline 12D, SEAM down, P6P7 & $0.4668(0.0096) ; 4$ & $0.6383(0.0655) ; 4$ \\
\hline 12D, SEAM down, P6 & $0.3650(0.0130) ; 3$ & $0.7275(0.0300) ; 3$ \\
\hline
\end{tabular}

* If we exclude 1 trajectory (the one that does not results in the full lateral break) then the Critical Breaking Force is: $0.6077(0.0290) ; 3$

\section{Table S3. Retraction pathways}

Pos - position of the indentation tip; $\boldsymbol{T} \_$name - type of MT lattice used; $\boldsymbol{I} \boldsymbol{p} \boldsymbol{I}$ - indentation plate (N)o/(Y)es; $\boldsymbol{I}$ fr - frame of indentation from which retraction was initiated; $\boldsymbol{I} \_\boldsymbol{t}$ - time of indentation after which retraction was initiated in ms; $\boldsymbol{R}_{-} \boldsymbol{f r}$ total numbers of retraction frames; $\boldsymbol{R} \_\boldsymbol{t}$ - total time of retraction in ms; $\boldsymbol{R} \_\boldsymbol{p l}$ - retraction with plate (N)o/(Y)es; $\boldsymbol{S} \_\boldsymbol{r}-$ full lateral and longitudinal recovery of the seam $(\mathrm{N}) \mathrm{o} /(\mathrm{Y}) \mathrm{es}$; $\mathbf{S r}$ _f - if seam fully recovered then at which frame of the retraction trajectory; $\boldsymbol{S r}_{-} \boldsymbol{t}$ - if seam fully recovered then at what time in ms of the retraction trajectory; $\boldsymbol{F}_{-}$rec - full recovery of all other interfaces; All trajectories that fail to recover are indicated in red.

\begin{tabular}{|c|c|c|c|c|c|c|c|c|c|c|c|}
\hline Pos. & $T \_$name & I_pl & I_fr & $\begin{array}{c}I \_t \\
{[m s]}\end{array}$ & $R \_f r$ & $\begin{array}{c}R_{-} t \\
{[\mathrm{~ms}]}\end{array}$ & $R \_p l$ & $\underset{c}{S \_r e}$ & Sr_fr & $\begin{array}{l}S r_{-} t \\
{[m s]}\end{array}$ & $F \_r e c$ \\
\hline \multirow{2}{*}{ p1 } & \multirow{2}{*}{ Reg } & \multirow{2}{*}{$N$} & 325 & 13.00 & 502 & 20.08 & $\mathrm{~N}$ & $\mathrm{Y}$ & 384 & 15.36 & Y \\
\hline & & & 375 & 15.00 & 502 & 20.08 & $\bar{N}$ & $\bar{Y}$ & 478 & 19.12 & $\bar{Y}$ \\
\hline \multirow{3}{*}{$\mathrm{p} 1$} & \multirow{3}{*}{ Reg } & \multirow{3}{*}{$\mathrm{N}$} & 260 & 10.40 & 502 & 20.08 & $\bar{N}$ & $\bar{Y}$ & 226 & 9.04 & $\bar{Y}$ \\
\hline & & & 330 & 13.20 & 502 & 20.08 & $\mathrm{~N}$ & $\mathrm{Y}$ & 357 & 14.28 & $\mathrm{Y}$ \\
\hline & & & 410 & 16.40 & 502 & 20.08 & $\mathrm{~N}$ & $\mathrm{~N}$ & & & $\mathrm{~N}$ \\
\hline \multirow{4}{*}{ p1 } & \multirow{4}{*}{$\mathrm{AHM}^{*}$} & \multirow{4}{*}{$Y$} & 200 & 8.00 & 256 & 10.24 & $\mathrm{~N}$ & $\bar{Y}$ & 0 & 0.00 & $\bar{Y}$ \\
\hline & & & 390 & 15.60 & 582 & 23.28 & $\mathrm{~N}$ & $Y$ & 0 & 0.00 & $Y$ \\
\hline & & & 200 & 8.00 & 365 & 14.60 & $Y$ & $Y$ & 0 & 0.00 & $Y$ \\
\hline & & & 390 & 15.60 & 573 & 22.92 & Y & Y & 0 & 0.00 & $\mathrm{~N}$ \\
\hline \multirow{2}{*}{$\mathrm{p} 1$} & \multirow{2}{*}{ Reg } & \multirow{2}{*}{ Y } & 300 & 12.00 & 510 & 20.40 & $\mathrm{~N}$ & $\mathrm{~N}$ & & & $\mathrm{~N}$ \\
\hline & & & 375 & 15.00 & 602 & 24.08 & $\mathrm{~N}$ & $\mathrm{~N}$ & & & $\mathrm{~N}$ \\
\hline \multirow{2}{*}{$\mathrm{p} 1$} & \multirow{2}{*}{ Reg } & \multirow{2}{*}{ Y } & 280 & 11.20 & 602 & 24.08 & $\mathrm{~N}$ & $Y$ & 225 & 9.00 & $Y$ \\
\hline & & & 360 & 14.40 & 602 & 24.08 & $\mathrm{~N}$ & $\mathrm{~N}$ & & & $\mathrm{~N}$ \\
\hline
\end{tabular}




\begin{tabular}{|c|c|c|c|c|c|c|c|c|c|c|c|}
\hline \multirow{2}{*}{$\mathrm{p} 2$} & \multirow{2}{*}{ Reg } & \multirow{2}{*}{$\mathrm{N}$} & 210 & 8.40 & 384 & 15.36 & $\mathrm{~N}$ & $Y$ & 102 & 4.08 & $Y$ \\
\hline & & & 400 & 16.00 & 502 & 20.08 & $\mathrm{~N}$ & $Y$ & 489 & 19.56 & $\bar{Y}$ \\
\hline \multirow{2}{*}{$\mathrm{p} 2$} & \multirow{2}{*}{$\mathrm{AHM}$} & \multirow{2}{*}{$Y$} & 190 & 7.60 & 256 & 10.24 & $\mathrm{~N}$ & $\bar{Y}$ & 140 & 5.60 & $\bar{Y}$ \\
\hline & & & 450 & 18.00 & 615 & 24.60 & $\mathrm{~N}$ & $Y$ & 399 & 15.96 & $Y$ \\
\hline \multirow{2}{*}{$\mathrm{p} 2$} & \multirow{2}{*}{ Reg } & \multirow{2}{*}{$Y$} & 215 & 8.60 & 475 & 19.00 & $\mathrm{~N}$ & $Y$ & 70 & 2.80 & $\bar{N}$ \\
\hline & & & 425 & 17.00 & 502 & 20.08 & $\mathrm{~N}$ & $\mathrm{~N}$ & & & $\mathrm{~N}$ \\
\hline \multirow{3}{*}{ p3 } & \multirow{3}{*}{ Reg } & \multirow{3}{*}{$\mathrm{N}$} & 210 & 8.40 & 502 & 20.08 & $\mathrm{~N}$ & $Y$ & 100 & 4.00 & $\mathrm{Y}$ \\
\hline & & & 360 & 14.40 & 502 & 20.08 & $\mathrm{~N}$ & $Y$ & 255 & 10.20 & $\bar{Y}$ \\
\hline & & & 450 & 18.00 & 502 & 20.08 & $\mathrm{Y}$ & $\mathrm{N}$ & & & $\mathrm{N}$ \\
\hline \multirow{2}{*}{ p3 } & \multirow{2}{*}{ Reg } & \multirow{2}{*}{$\mathrm{N}$} & 245 & 9.80 & 502 & 20.08 & $\mathrm{~N}$ & $Y$ & 170 & 6.80 & $\bar{Y}$ \\
\hline & & & 435 & 17.40 & 502 & 20.08 & $\mathrm{~N}$ & $\mathrm{~N}$ & & & $\mathrm{~N}$ \\
\hline \multirow{2}{*}{ p3 } & \multirow{2}{*}{$\mathrm{AHM}$} & \multirow{2}{*}{$Y$} & 240 & 9.60 & 354 & 14.16 & $\mathrm{~N}$ & $Y$ & 120 & 4.80 & $\bar{Y}$ \\
\hline & & & 450 & 18.00 & 615 & 24.60 & $\mathrm{~N}$ & $\mathrm{~N}$ & & 0.00 & $\mathrm{~N}$ \\
\hline \multirow{2}{*}{ p3 } & \multirow{2}{*}{ Reg } & \multirow{2}{*}{$Y$} & 290 & 11.60 & 440 & 17.60 & $\mathrm{Y}$ & $Y$ & 125 & 5.00 & $\mathrm{~N}$ \\
\hline & & & 415 & 16.60 & 502 & 20.08 & $Y$ & $Y$ & 498 & 19.92 & $\mathrm{~N}$ \\
\hline \multirow{2}{*}{ p4 } & \multirow{2}{*}{ Reg } & \multirow{2}{*}{$\mathrm{N}$} & 240 & 9.60 & 502 & 20.08 & $\mathrm{~N}$ & $Y$ & 167 & 6.68 & $Y$ \\
\hline & & & 410 & 16.40 & 502 & 20.08 & $\mathrm{~N}$ & $Y$ & 279 & 11.16 & $Y$ \\
\hline \multirow{2}{*}{$\mathrm{p} 4$} & \multirow{2}{*}{$\mathrm{AHM}$} & \multirow{2}{*}{$Y$} & 230 & 9.20 & 439 & 17.56 & $\mathrm{~N}$ & $Y$ & 99 & 3.96 & $Y$ \\
\hline & & & 400 & 16.00 & 502 & 20.08 & $\mathrm{~N}$ & $\mathrm{~N}$ & & & $\mathrm{~N}$ \\
\hline \multirow{2}{*}{$\mathrm{p} 4$} & \multirow{2}{*}{ Reg } & & 290 & 11.60 & 440 & 17.60 & $\mathrm{Y}$ & $Y$ & 185 & 7.40 & $\mathrm{~N}$ \\
\hline & & $Y$ & 430 & 17.20 & 502 & 20.08 & $\mathrm{~N}$ & $Y$ & 488 & 19.52 & $\mathrm{~N}$ \\
\hline$n 5$ & Reg & $N$ & 220 & 8.80 & 502 & 20.08 & $\mathrm{~N}$ & $Y$ & 193 & 7.72 & $Y$ \\
\hline o & neg & T & 370 & 14.80 & 502 & 20.08 & $\mathrm{~N}$ & $\mathrm{~N}$ & & & $\mathrm{~N}$ \\
\hline & & & 225 & 9.00 & 396 & 15.84 & $\mathrm{~N}$ & $Y$ & 210 & 8.40 & $Y$ \\
\hline 05 & $\mathrm{AHM}$ & Y & 330 & 13.20 & 602 & 24.08 & $\mathrm{~N}$ & $\mathrm{~N}$ & & & $\mathrm{~N}$ \\
\hline ק & HITा & 1 & 225 & 9.00 & 331 & 13.24 & $\mathrm{Y}$ & $Y$ & 190 & 7.60 & $\bar{Y}$ \\
\hline & & & 330 & 13.20 & 598 & 23.92 & $Y$ & $\mathrm{~N}$ & & & $\mathrm{~N}$ \\
\hline p5 & Reg & $Y$ & 320 & 12.80 & 502 & 20.08 & $Y$ & $Y$ & 343 & 13.72 & $Y$ \\
\hline po & neg & $\mathrm{r}$ & 410 & 16.40 & 502 & 20.08 & $\mathrm{Y}$ & $\mathrm{N}$ & & & $\mathrm{N}$ \\
\hline
\end{tabular}

* initially the seam was not broken, lateral break was between P12/P13 


\section{Table S4. Thermodynamics}

Thermodynamic measures from each indentation/retraction run and for each indentation point near the seam, calculated as described in the main text. Additionally, for each trajectory we calculated the Gibbs Free energy barrier only for the portion of a run corresponding to the lateral break of the seam $\left(\Delta G_{\text {SEAM }}\right)$. This is the area under the indentation force versus indentation depth plot corresponding to the frames where the first lateral break of the dimers at the seam occurs up to the frame at which retraction was initiated (gray boxes depicted in Figure.S12). Then we calculated the contribution of $\Delta G_{\text {SEAM }}$ to the $\Delta G$ entry from the second column as the average percentage contribution for each indentation point, as well as for all indentation points taken together. "\# of samples" refers to the number of different runs used to calculate the thermodynamic values for each indentation position.

\begin{tabular}{c|r|r|r|r|r} 
Position & \multicolumn{1}{c|}{$\begin{array}{c}\Delta \mathrm{G} \\
{[\mathrm{kcal} / \mathrm{mol}]}\end{array}$} & $\begin{array}{c}\Delta \mathrm{H} \\
{[\mathrm{kcal} / \mathrm{mol}]}\end{array}$ & $\begin{array}{c}\mathrm{T} \Delta \mathrm{S} \\
{[\mathrm{kcal} / \mathrm{mol}]}\end{array}$ & $\begin{array}{r}\Delta \mathrm{G}_{\mathrm{SEAM}} \\
{[\%]}\end{array}$ & $\#$ of samples \\
\hline $\mathrm{p} 1$ & 5.504 & 15.374 & 9.870 & 15.88 & 2 \\
p2 & 3.749 & 13.318 & 9.569 & 19.79 & 1 \\
p4 & 4.236 & 15.531 & 11.294 & 21.73 & 3 \\
p5 & 5.615 & 16.959 & 11.343 & 15.08 & 2 \\
AVG & 4.623 & 14.147 & 9.525 & 14.81 & 2 \\
ST DEV & 4.746 & 15.066 & 10.320 & 17.46 & \\
\hline AVG* & 0.806 & 1.396 & 0.921 & 3.12 & \\
ST DEV & 4.995 & 15.503 & 10.508 & 16.87 & \\
\hline
\end{tabular}

* Average for position p1, p3, p4 and p5

\# The lowest and highest contributions for individual trajectories are respectively: $11.53 \%$ (p5) and 25.65\% (p3). 


\section{Supplemental Figures}

Fig.S1.A MT structure, protofilament labeling.

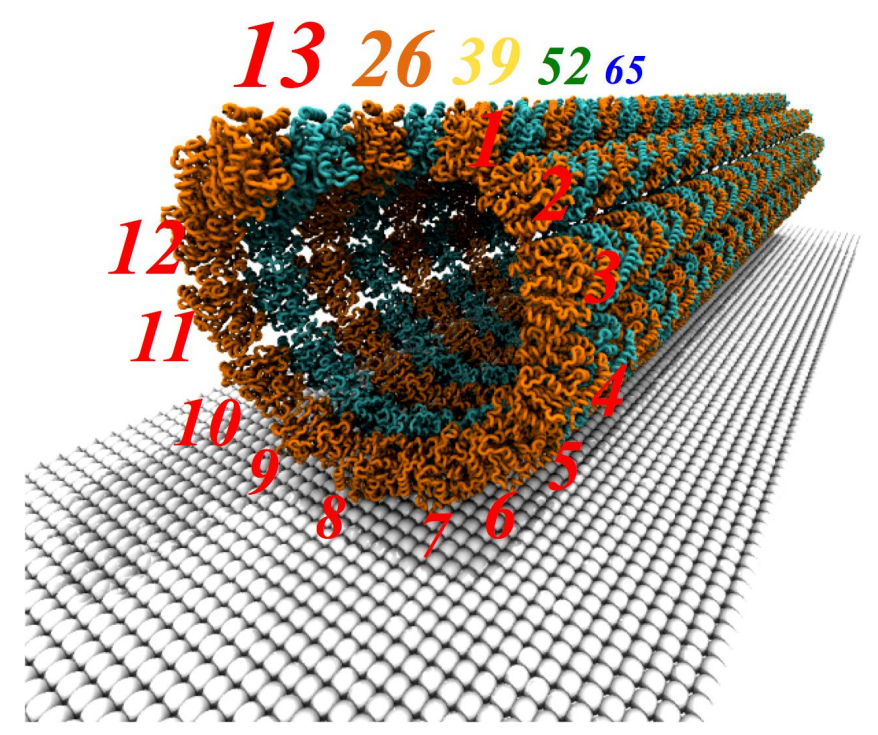

We considered only 13 protofilaments MTs. The numbering began at the alpha end (orange, minus) with the monomer that is inset along the seam, and proceeded clockwise. We placed sets of 26 monomers, or rings of 13 heterodimers, longitudinally (beta to alpha) in order to construct the 8D, 12D, and 16D length MT filaments.

Fig.S1.B Cartoon representation of control and high-salt MTs used in the severing assays.

\section{A. Control Microtubules}

12-13 protofilaments, B-lattice

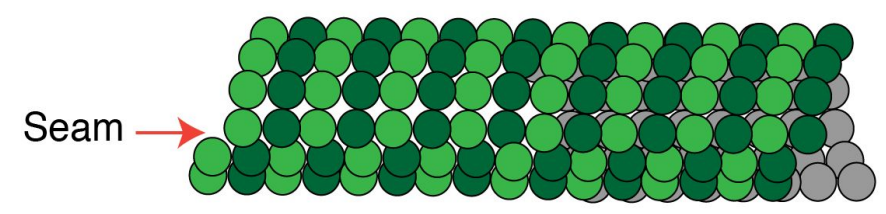

\section{B. Seam-Defect Microtubules}

10 protofilaments, mixed $A$ - and B-lattices

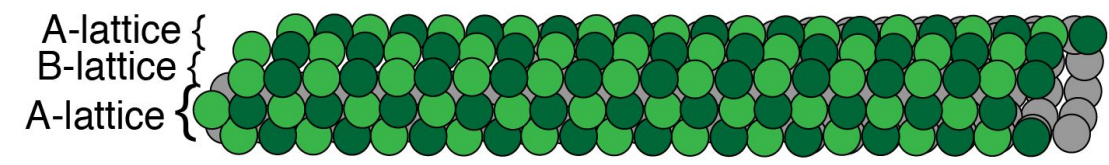

Panel A shows the cartoon of a regular MT lattice with only one seam (A-lattice interface). Panel B shows a cartoon of a high-salt MT with $50 \%$ seams. 


\section{Fig.S2. Protofilament Bending Angle for MTs with Both Ends Fixed}
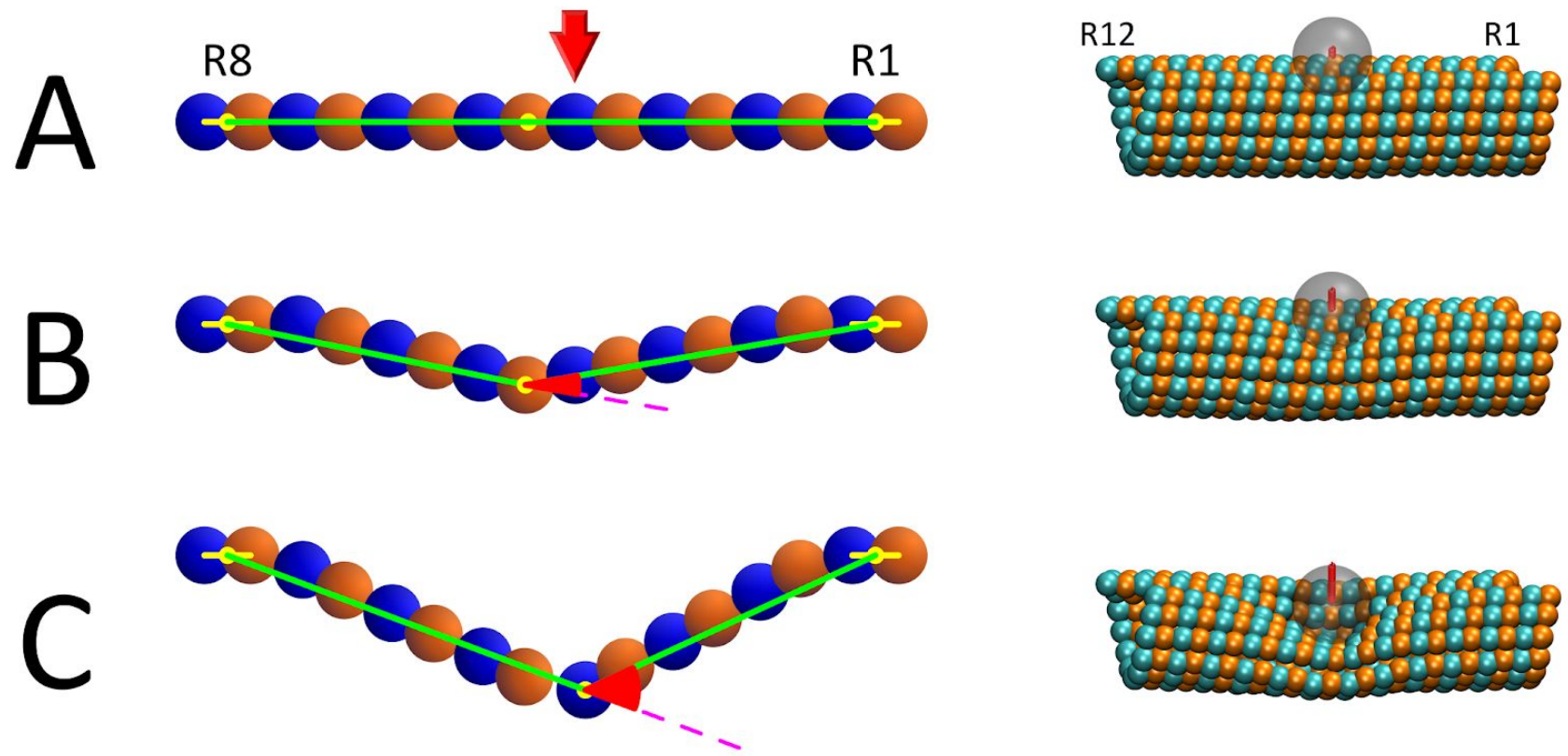

Schematics for angles measurements (left) and example snapshots from a 12D MT indentation trajectory (right). Orange and blue balls represent the alpha and beta tubulin monomers; the red arrow indicates the initial indentation point, and the red angle is the measured angle which we compare with the experimental data. A the initial configuration; B - a configuration with visible bending of the protofilament; $\mathrm{C}$ - a configuration with a longitudinal break in the protofilament. 


\section{Fig.S3. Protofilament Bending Angle for MTs with the Plus End Free}
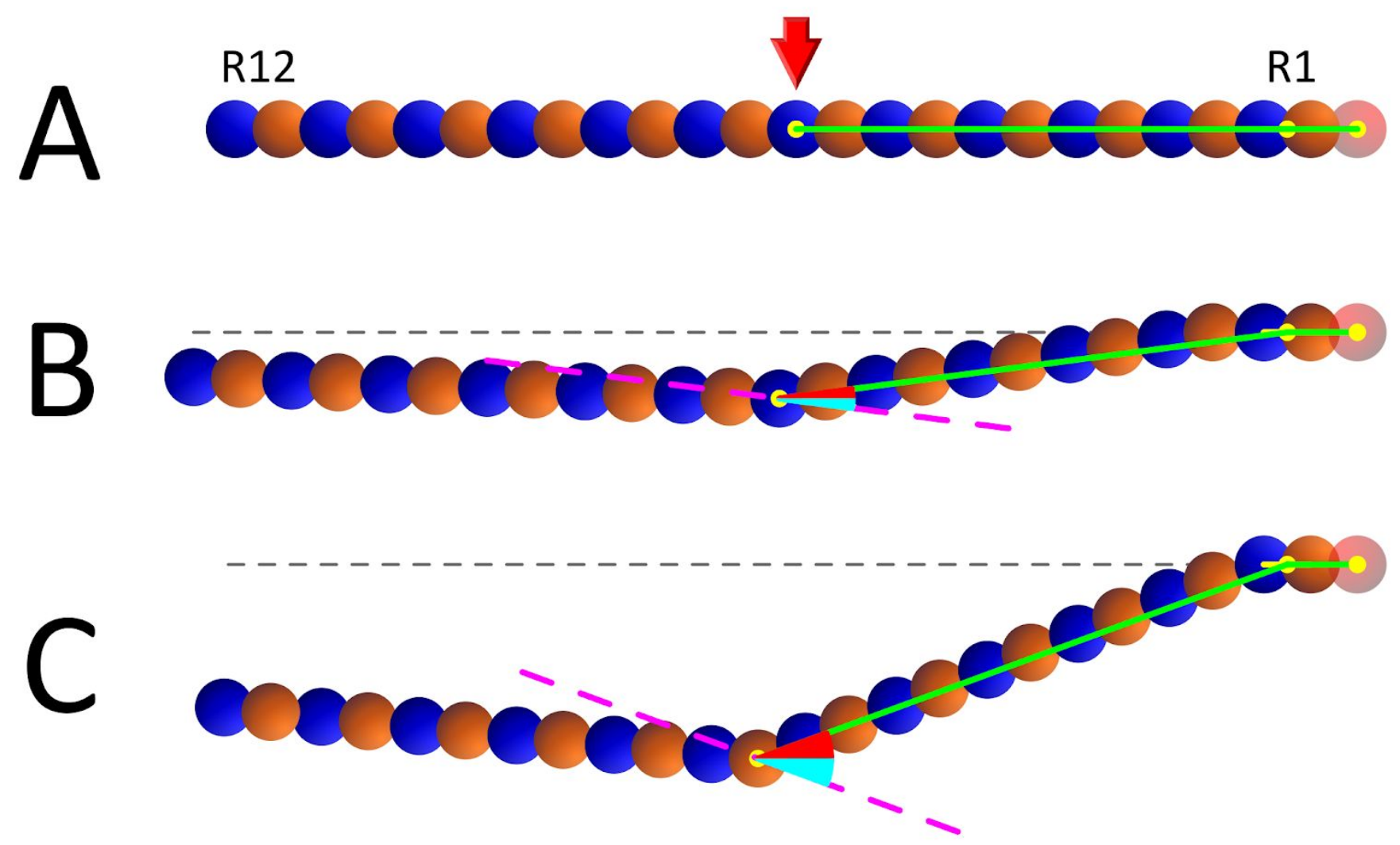

For the simulations with the plus end free because we released the constraints at the plus end previously frozen dimers are now able to move. Therefore any measurements with the method described for ends fixed in MTs longer than 8D led to underestimated bending angles. For the 8D case we did not observe this effect due to the cancellation of two effects: 1) on the minus side we overestimate the measured angles because of the biased end; and 2) on the plus end we underestimate the angles. For the longer 12D, and 16D MTs the movement of the dimers from the plus end led to an additional extension of the angle arm (from the center of the system to the plus end), which caused the underestimation of the angle on the plus side. Thus, for the 12D polymer with plus free end case we measure the angle between the following points: 1) the center of mass of the frozen dimer (first dimer on the minus end) shifted toward the minus end by $50 \AA ; 2$ ) the center of mass of the frozen dimer; and 3) the center of mass of the next monomer in the PF. However because this measurement accounts only for the left-hand "half" side of the MT we had to multiply this angle by 2 . Then we follow a similar procedure to the one described earlier: for each frame and for each PF we select the angle with the highest absolute value. 
Fig.S4. Evolution of Force, Native Contacts, and Protofilament Bending Angle.

A

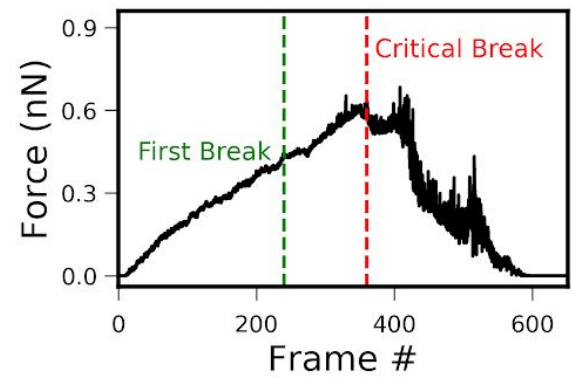

B

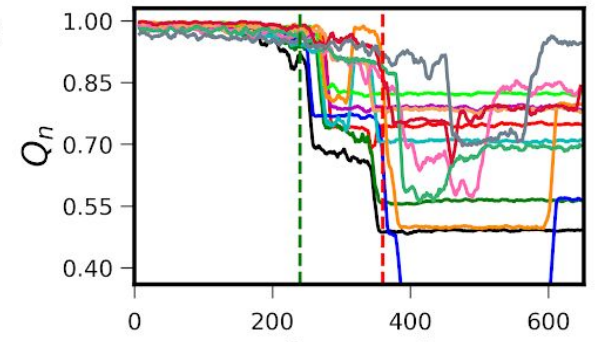

Frame \#
C

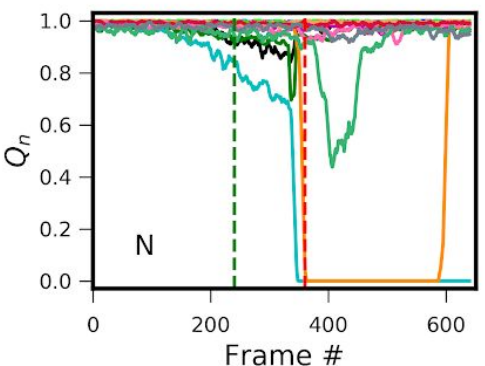

D

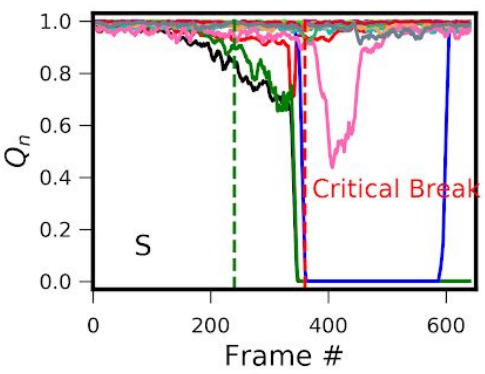

E

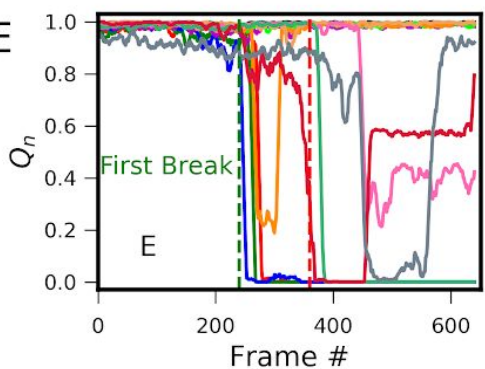

$\mathrm{F}$



G

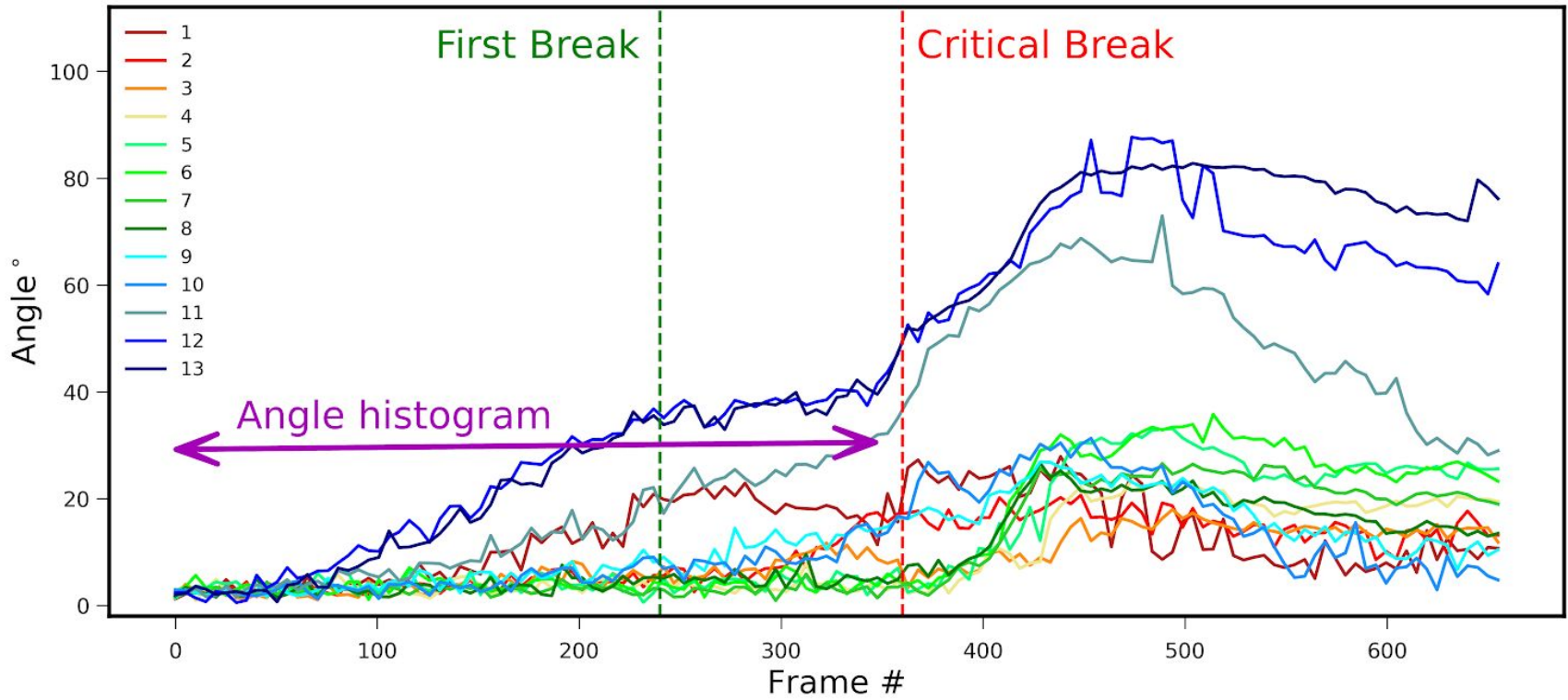

The time evolution of the force, native contacts, and protofilament bending angle for indentation on position 1. The force indentation, contact analysis as $Q_{n}$ overall, north $(N)$, south $(S)$, east $(E)$, and west (W) monomer interface, and the bending angles by PF. The histograms for the first and critical breaking forces were constructed from the force indentation curve at the green dashed line (first) and red dashed line (critical), as determined by the breaking of the lateral contacts, seen in the western and eastern interfaces (first) and north and south interface (critical), respectively.
A. The force versus frame curve for position 1.
B-F. $Q_{n}$ overall, and for each monomer interface.

G. The PF bending angle. 
Fig.S5. Force versus indentation depth for pushing on the seam

A



B

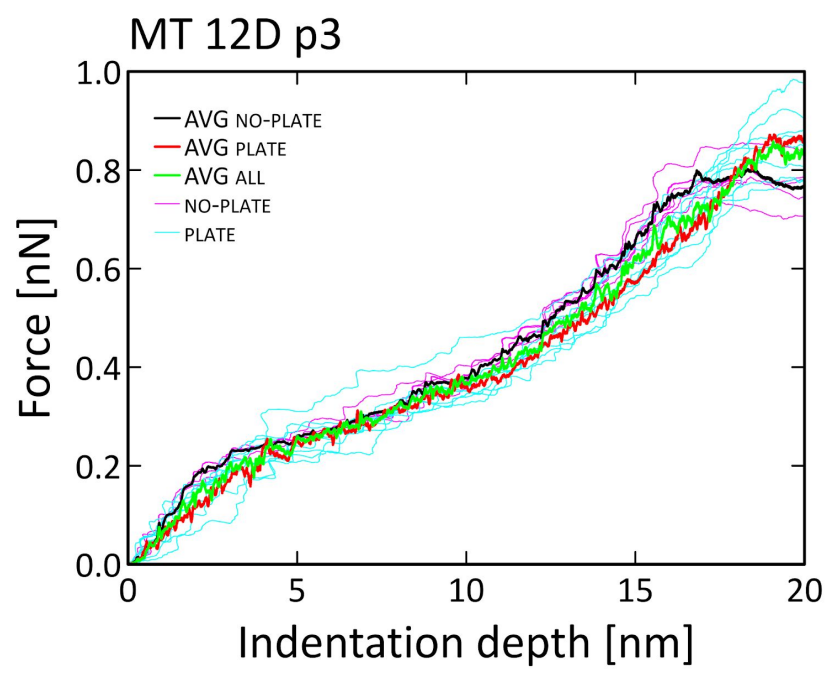

An example of the indentation forces vs. indentation depth (for all trajectories without the plate - black line; with the plate - red line and all trajectories together - green line) for indentation on position p3 (between PFs P1 and P13) for MT 8D (A) and MT 12D (B) systems with both ends fixed. Magenta - all trajectories without plate; cyan - trajectories with the plate. Here we show the data from all trajectories, as well as the respective averages.

\section{Fig.S6. The average indentation forces for trajectories with the plus end free}

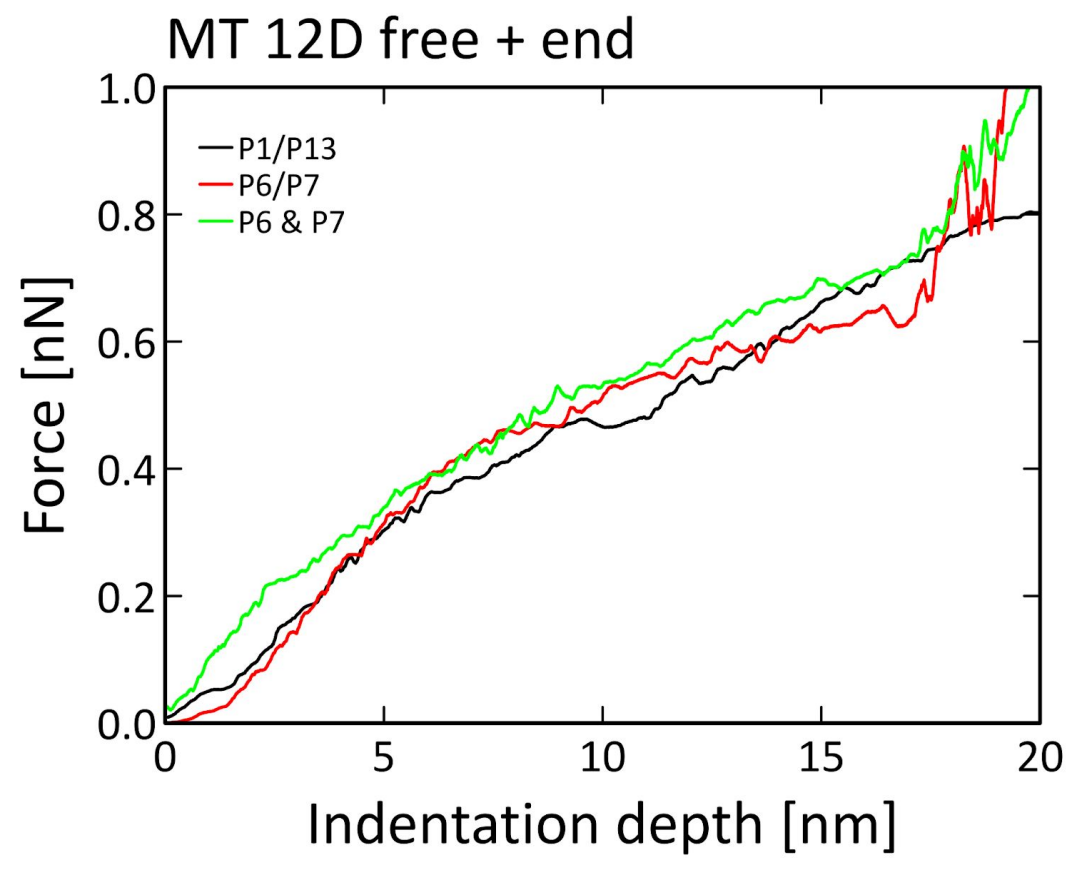

The average indentation forces vs. indentation depth for trajectories with the plus end free: for indentation on the seam (between PFs P1 and P13) - black line; between PFs P6 and P7 - red line; and for the combined data from indentation on either one of the PFs: P6 and P7 - green line. 
Fig.S7. Distributions of first breaking force by MT length and indentation position.
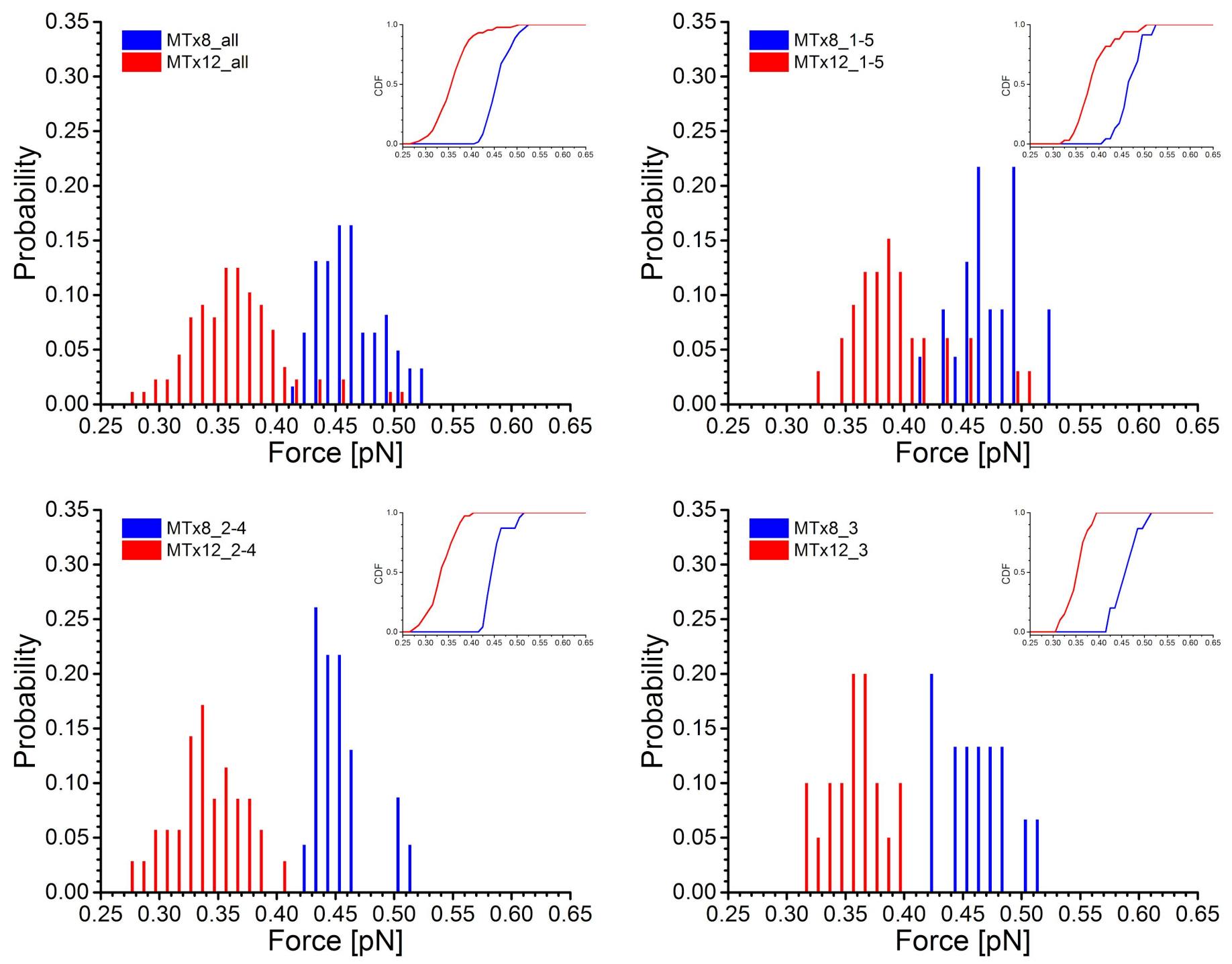

The first breaking forces were categorized by position (pos-1,5, pos-2,4, and pos-3). The Kolmogorov-Smirnov test was employed to determine if the distributions were equivalent. As the polymer length increased, the distribution of the first breaking forces shifted to lower forces, resulting in different distributions between the $8 \mathrm{D}$ (blue) and 12D (red) polymers. The top left plot shows the collective data from all indentation positions for the MT $8 \mathrm{D}$ versus the $12 \mathrm{D}$ filaments. The top right shows the distribution for the indentation on $\mathrm{p} 1$ and $\mathrm{p} 5$. The bottom left shows the distributions for the indentation on $\mathrm{p} 2$ and $\mathrm{p} 4$. The bottom right shows the distributions for the indentation on $\mathrm{p} 3$ (the seam). The insets show the corresponding cumulative distribution functions (CDFs). 


\section{Fig.S8. Native vs. Non-native contacts}

The time evolution of the native and non-native contacts for the MT 8D and MT 12D on various positions. The $y$-axis reports the Relative $Q_{n}$, which is the total number of contacts (native or non-native) for an interface at each frame divided by the average value of the native contacts for that type of interface (lateral, longitudinal or the seam) in the starting configuration. For the left panel (the seam): green - P1 longitudinal contacts, blue P1/P13 lateral contacts, red - P13 longitudinal contacts; for the middle panel P5: green - P5 longitudinal contacts, magenta - P5/P6 lateral contacts, cyan - P4/P5 - lateral contacts; for the right panel: P12: green - P12 longitudinal contacts, magenta - P12/P13 lateral contacts, cyan - P11/P12 - lateral contacts; P2: green - P2 longitudinal contacts, magenta - P2/P3 lateral contacts, cyan - P1/P2 - lateral contacts; P6/P7: green - P6 longitudinal contacts, blue - P6/P7 lateral contacts, red - P7 longitudinal contacts.

A. MT 12D both ends fixed indentation on position p3 (between protofilaments P1/P13)
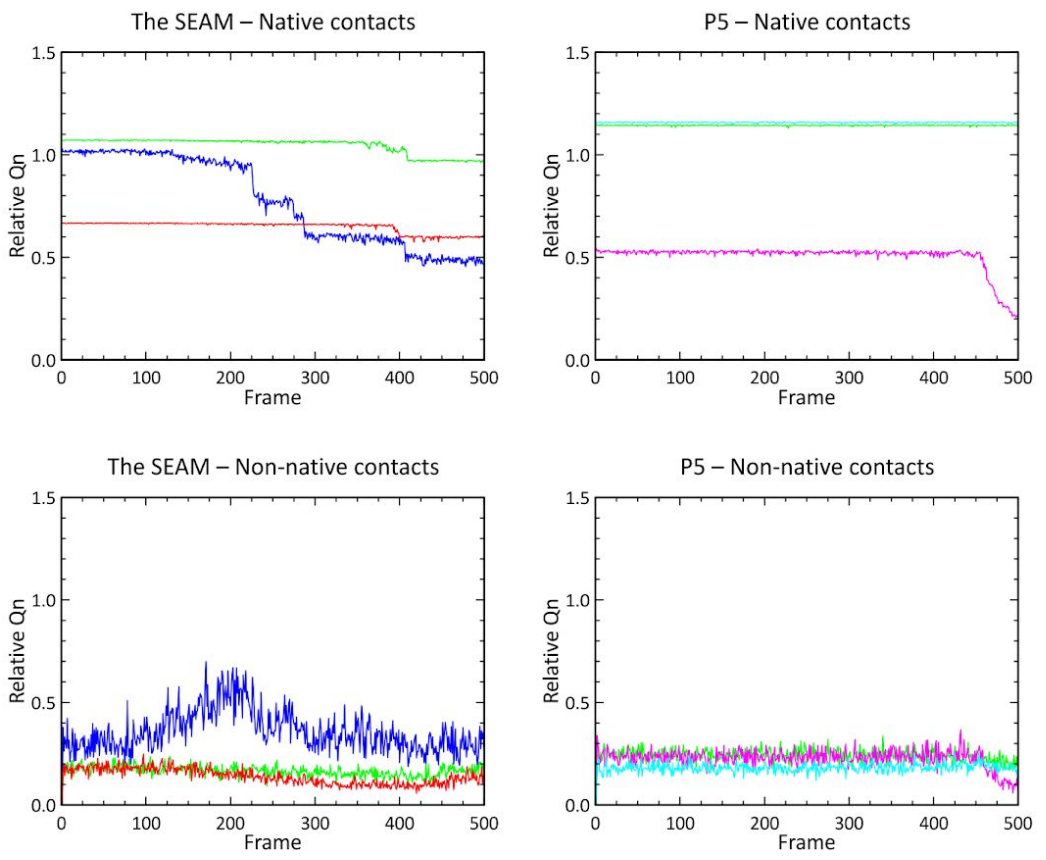

B. MT 12D indentation on position p1 (between protofilaments P12/P13)
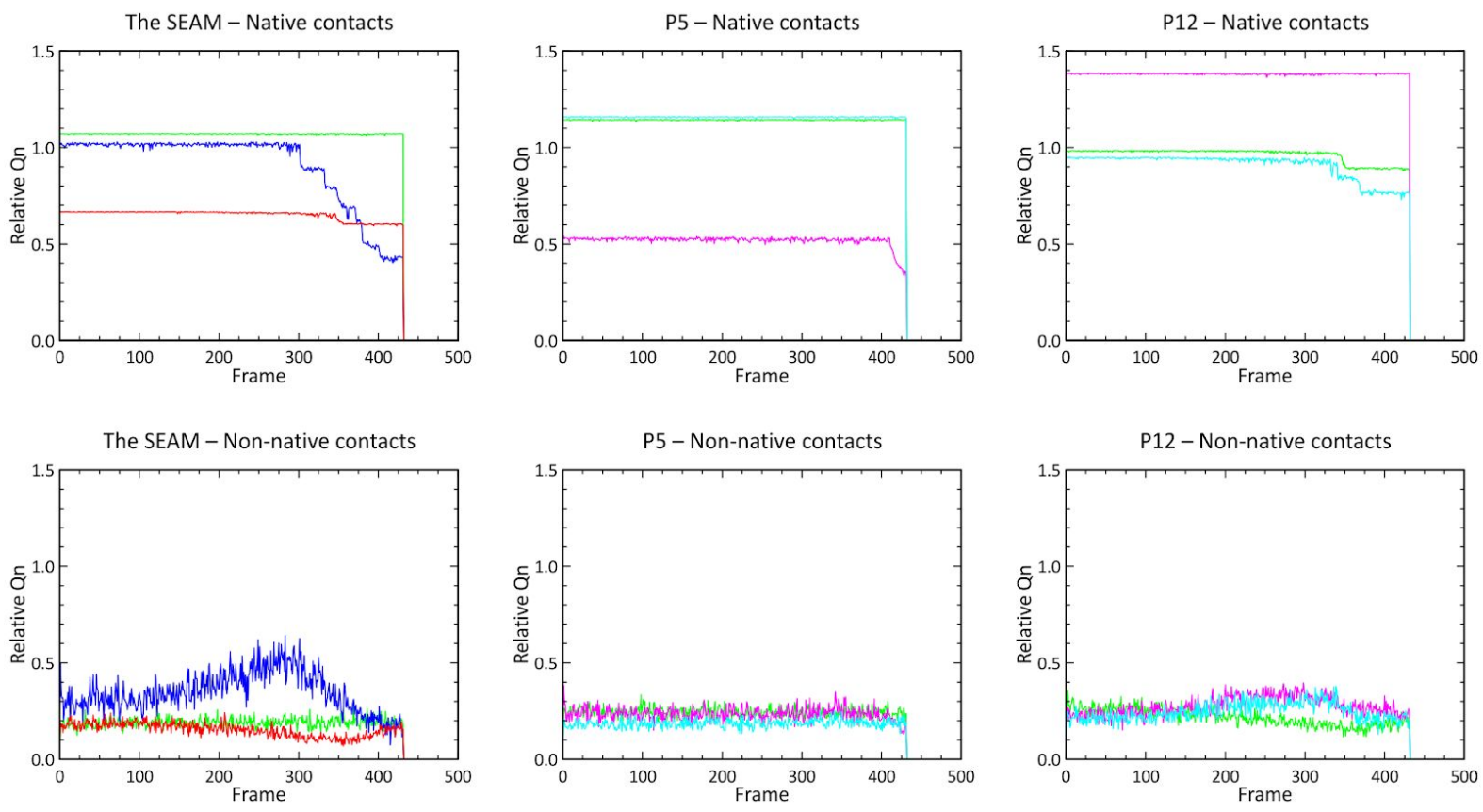
C. MT 12D both ends fixed indentation on position p5 (between protofilaments P1/P2)
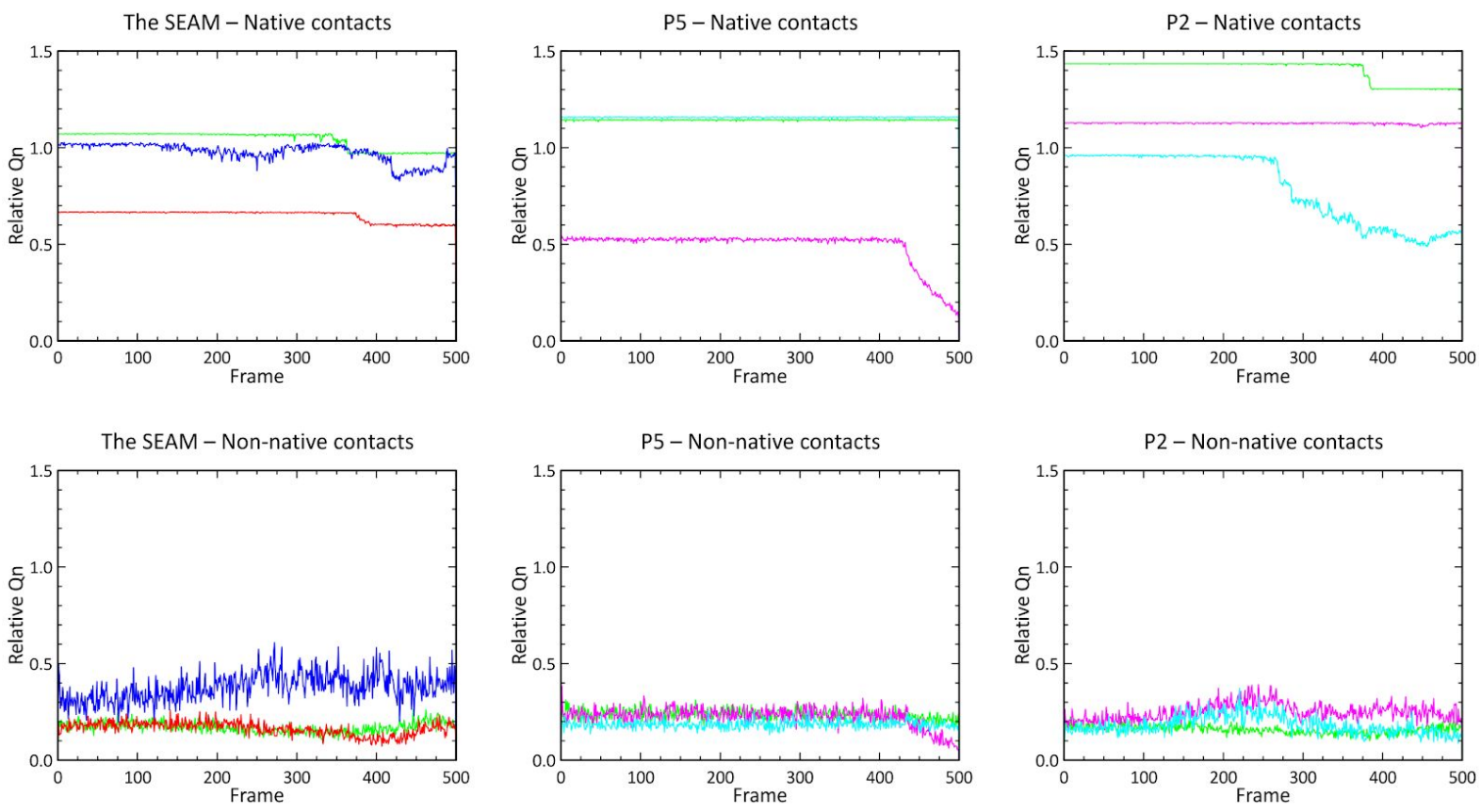

D. MT 12D both ends fixed indentation on position p2 (on protofilament P13)
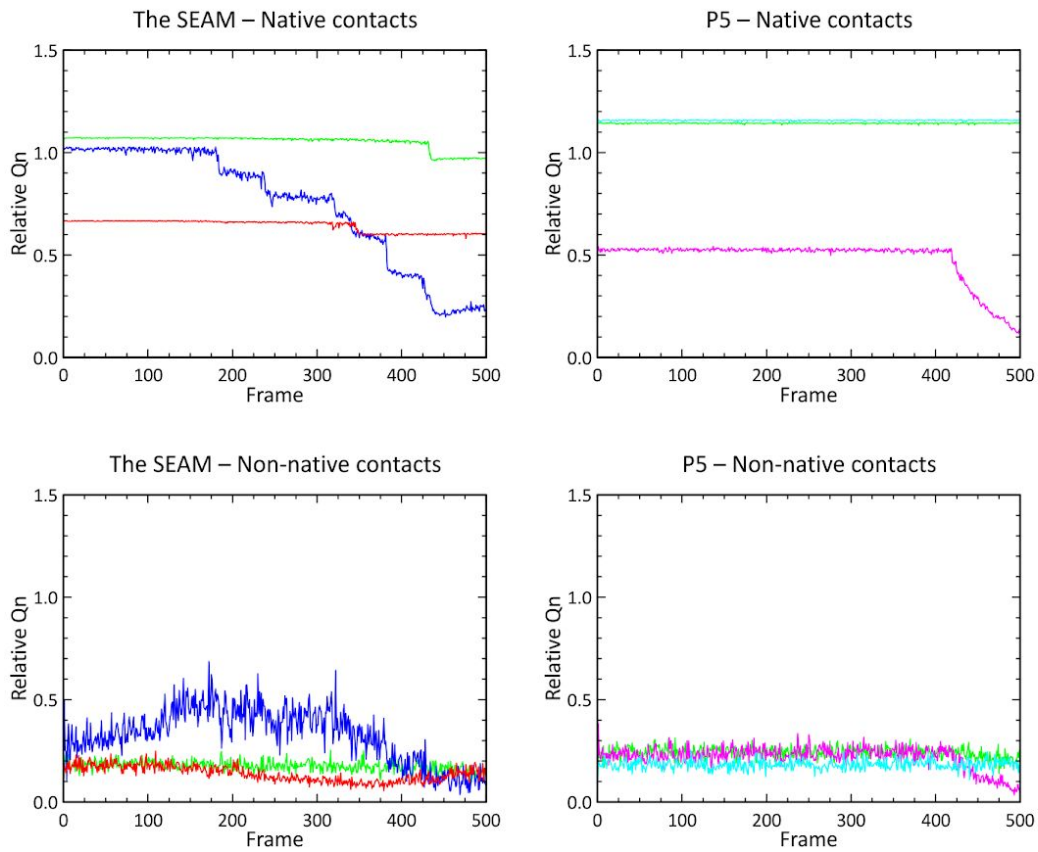


\section{E. MT 12D both ends fixed indentation on position p4 (on protofilament P1)}
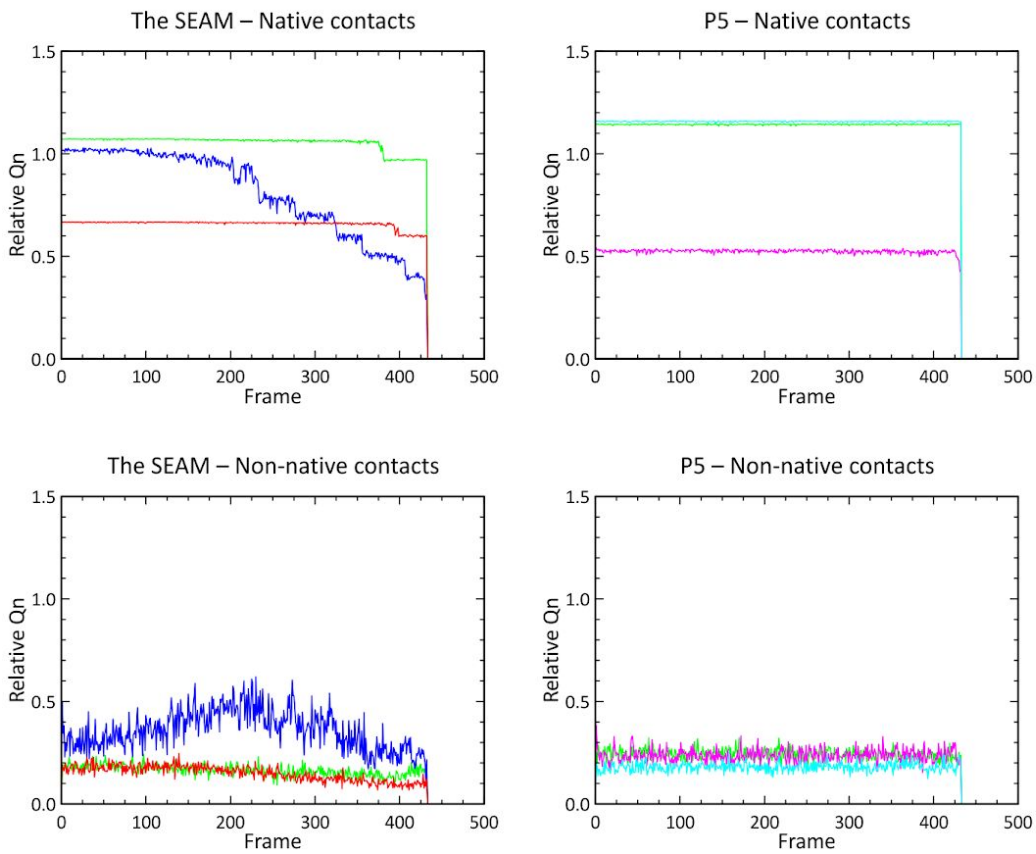

F. MT 12D with free plus end indentation between protofilaments P6/P7
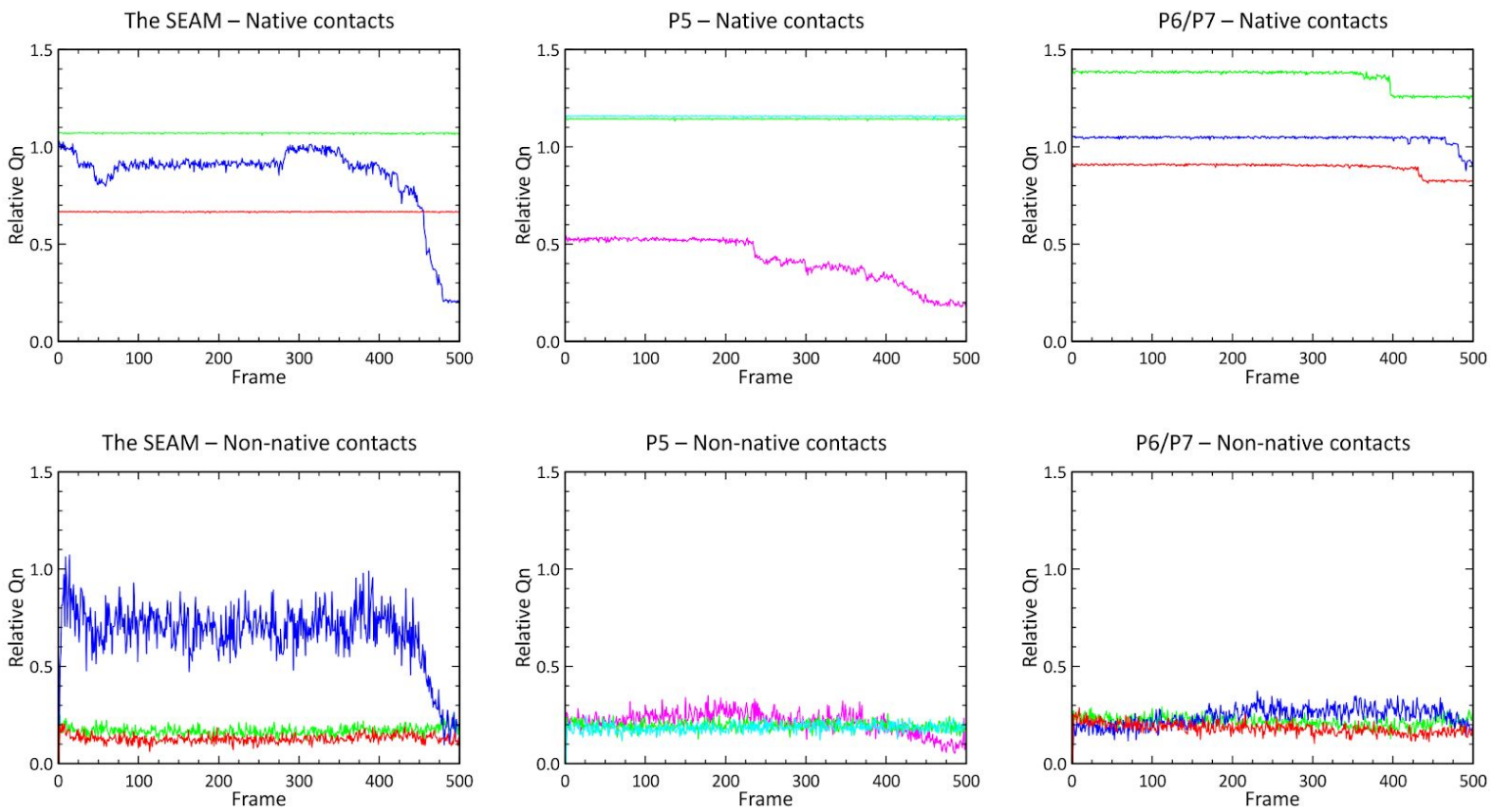
G. MT 12D with free plus end indentation on position p3 (between protofilaments P1/P13)
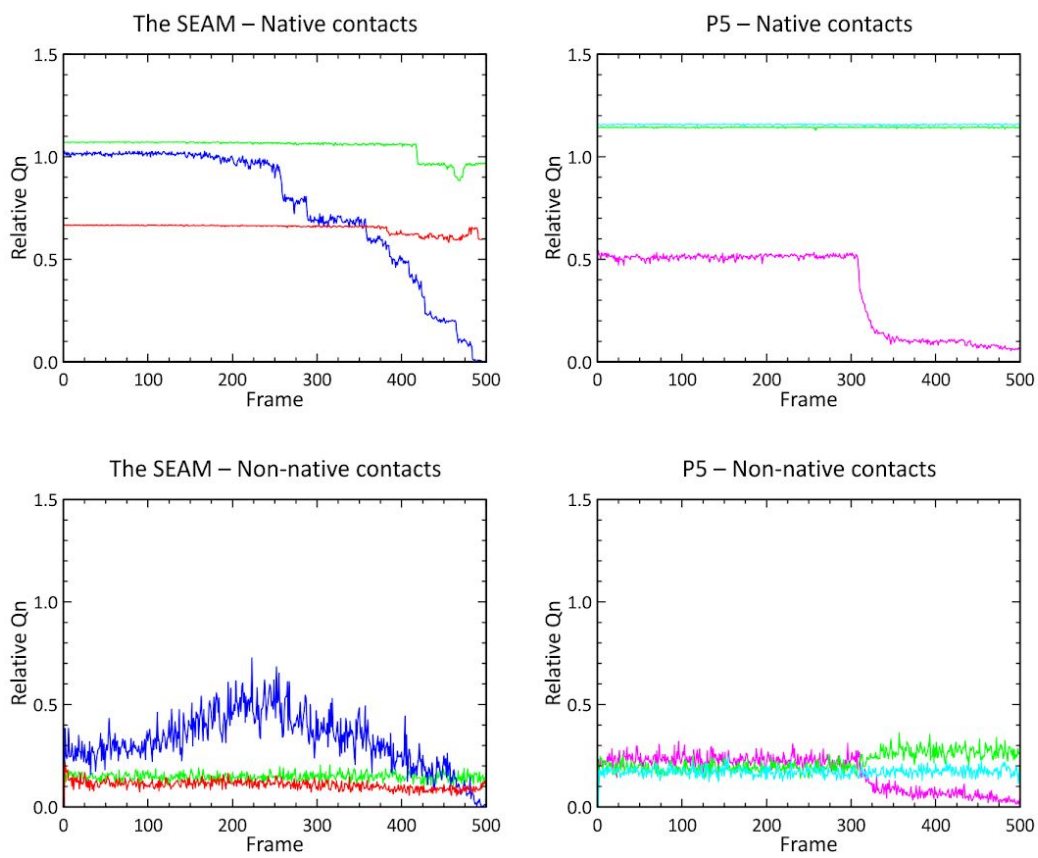

H. MT 8D both ends fixed indentation between protofilaments P6/P7
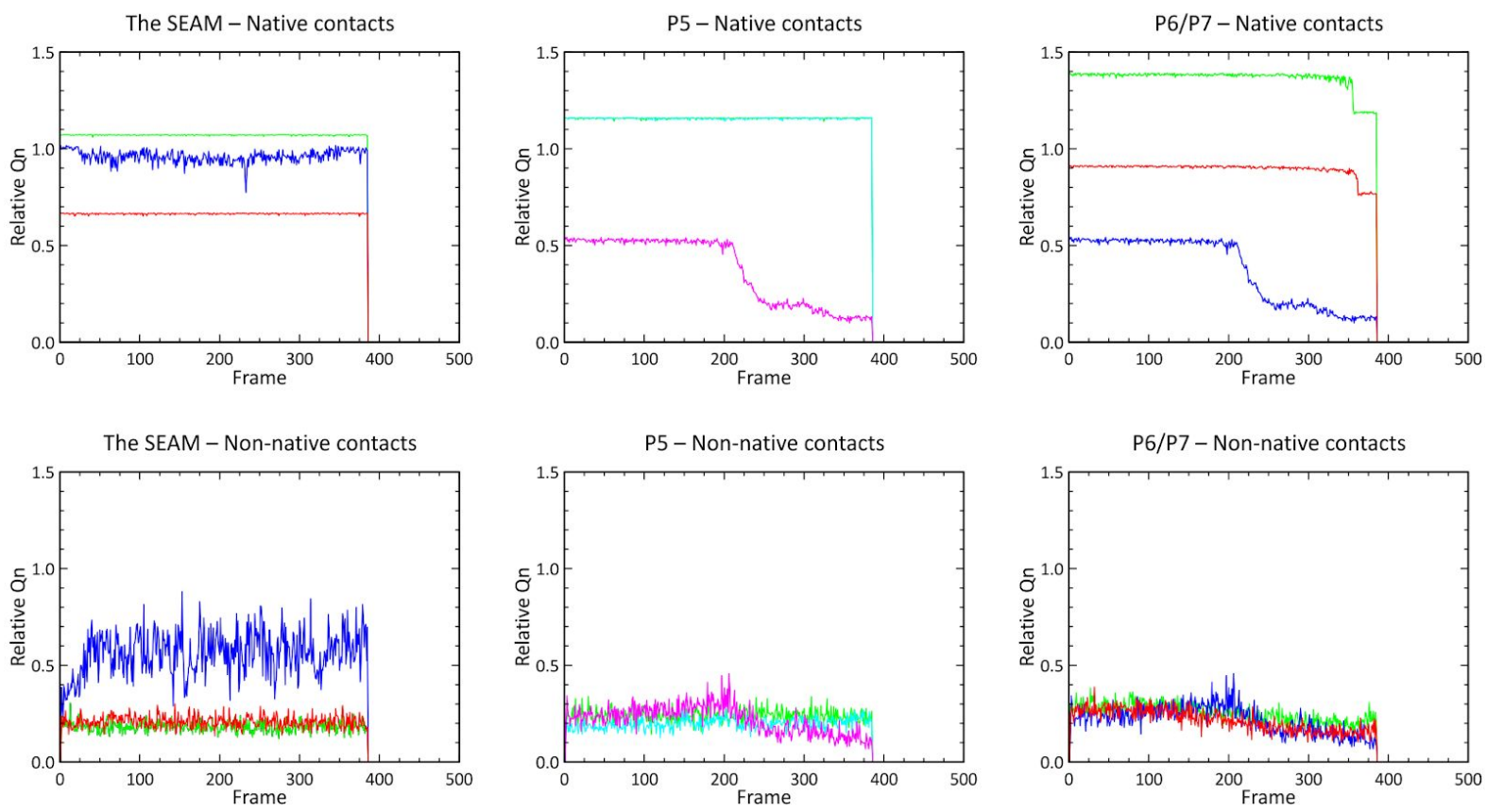

\section{Fig.S9. Snapshots of representative states along the major indentation pathways for the MT 12D filaments.}

In all views, $f$ stands for the frame number in the simulations. The indentation region is circled in black and the 
actual subunits pushed are colored differently from the rest of the lattice (in red and/or light green).

A. MT 12D, p1p5, both ends fixed

View from: $A$ - minus end; $B$ - plus end; $C$ - from the side minus -> plus; $D$ - from the top minus -> plus fO

$f 200$

A

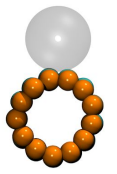

B

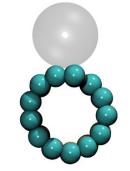

A

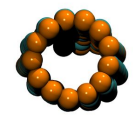

B

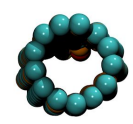

A



C

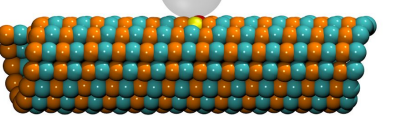

D $\quad B$



$f 500$

A

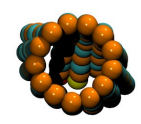

B

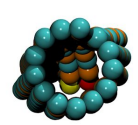

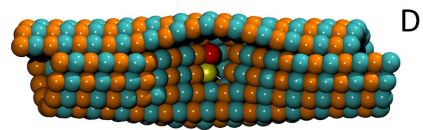

C

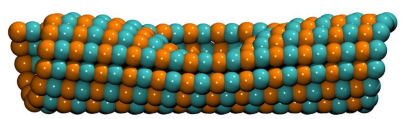

D

\section{D}

B

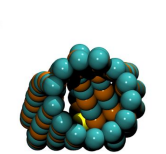

B. MT 12D, p3, both ends fixed

View from: A - minus end; $B$ - plus end; C - from the side minus -> plus; D - from the top minus -> plus

fO

A

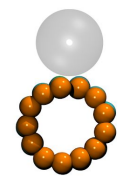

B

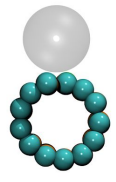

A

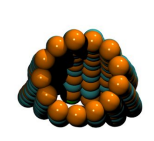

B

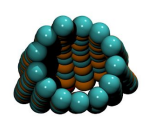

C
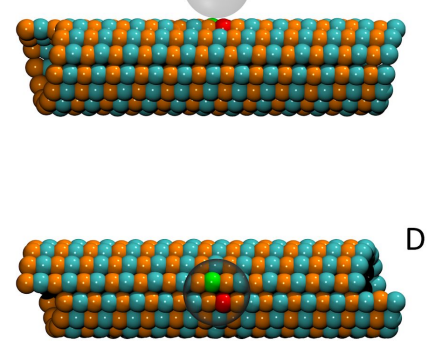

f425
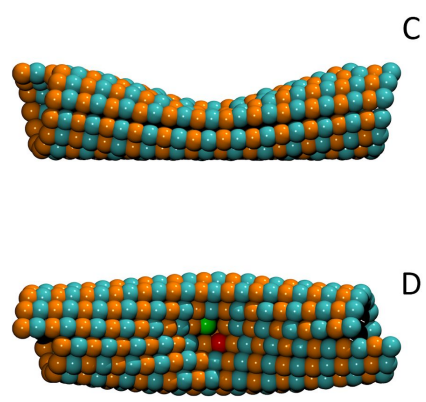

A

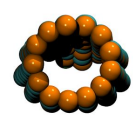

B

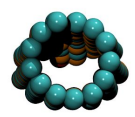

A

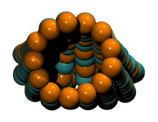

B

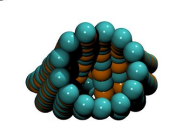

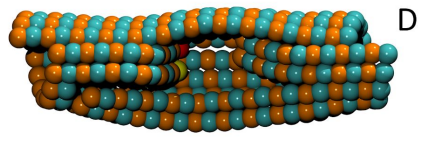

f200

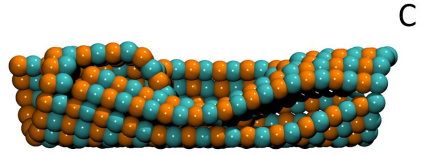

C
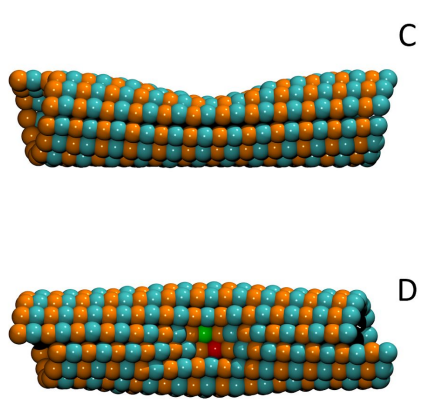

f500
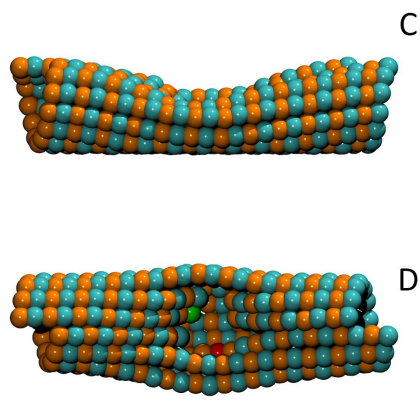
C. MT 12D, p3, plus end free, pathway without propagation to the plus end

View from: A - minus end; $B$ - plus end; C - from the side minus -> plus; $D$ - from the top minus -> plus fO

$f 200$

A

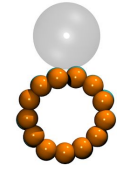

B

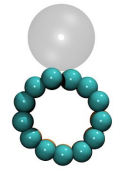

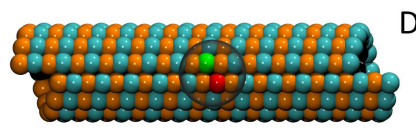

f375
A

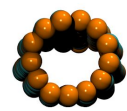

B

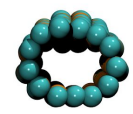

A

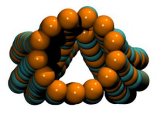

C
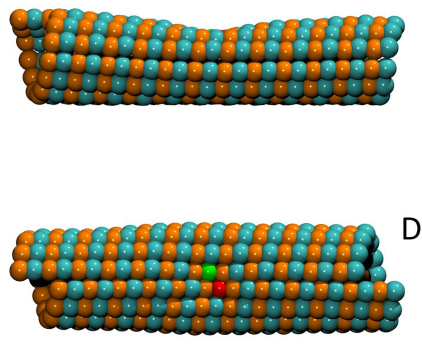

$f 475$
A

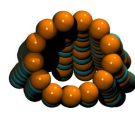

B

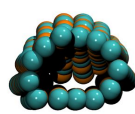

C
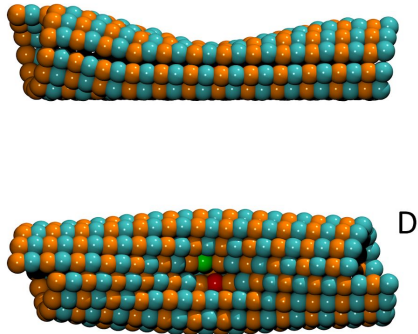

D

B

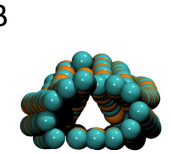

$\mathrm{C}$
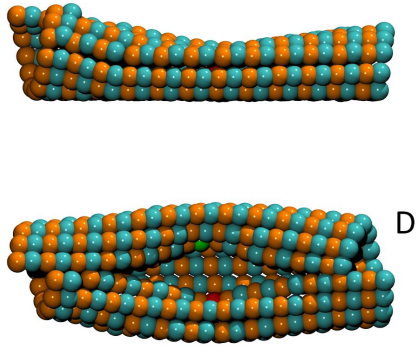

D. MT 12D, P6/P7, plus end free; trajectory without propagation to the plus end

View from: A - minus end; B - plus end; $C$ - from the side minus -> plus; $D$ - from the top minus -> plus

fO

A

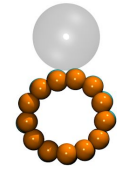

B

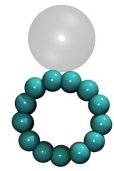

C
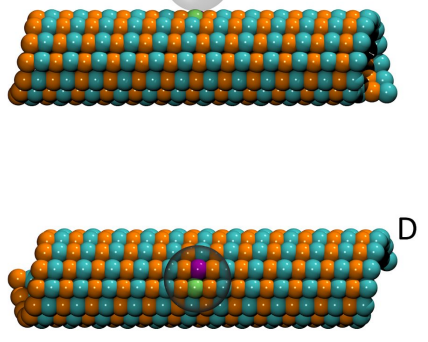

f400 f200

A

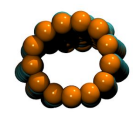

B

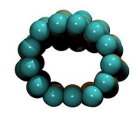

A

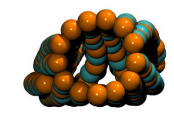

B

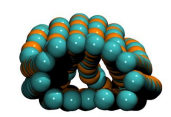

C
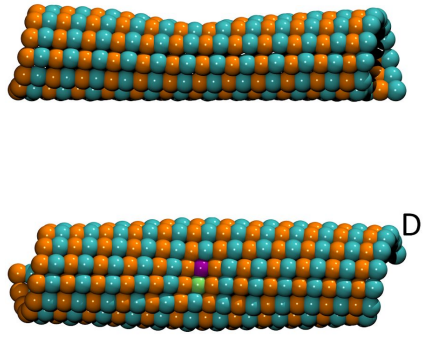

f550
A

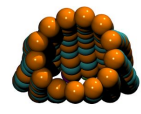

B

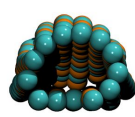

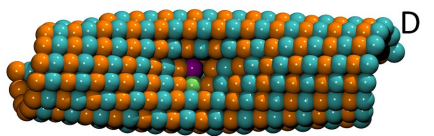

C

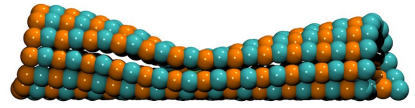


E. MT 12D, P6, plus end free

View from: $A$ - minus end; $B$ - plus end; $C$ - from the side minus -> plus; $D$ - from the top minus -> plus fO

f200

A

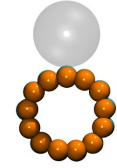

B

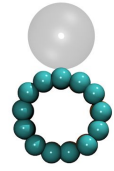

A

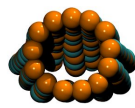

B



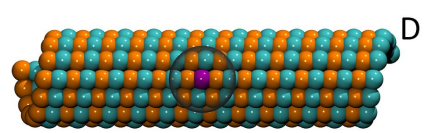

f375
A

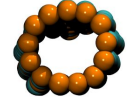

B
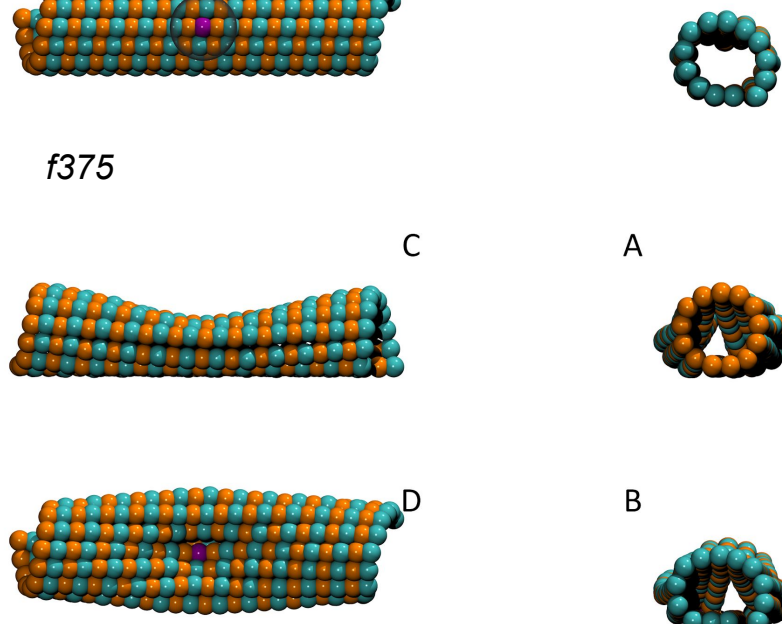

A



B

C
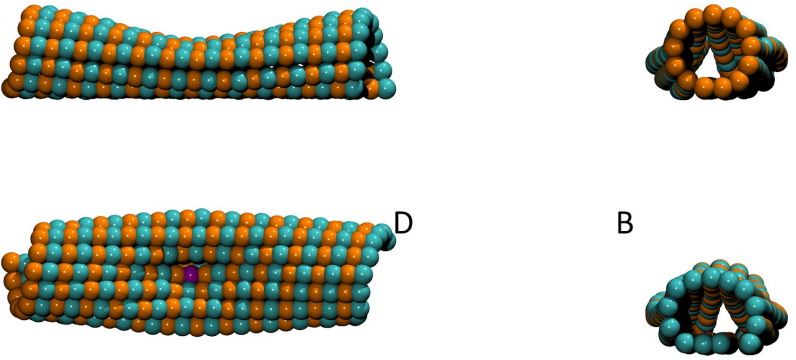

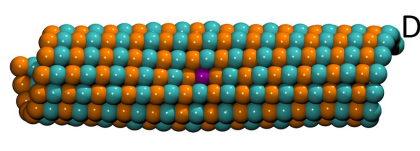

f497

C

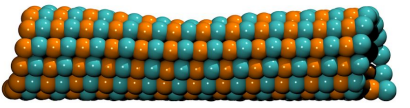

C
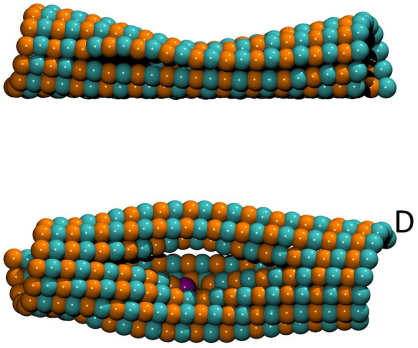


\section{Fig.S10. Results of the KS test for the bending angles}

We carried out the KS test for the cumulative distribution functions (CDF) from our various types of indentation simulations vs. the control and the high salt results from experiments. KS test was performed in Origin Pro. This figure depicts the resulting log-log plots of the probability (sample vs. control) vs. (sample vs. high salt).

A

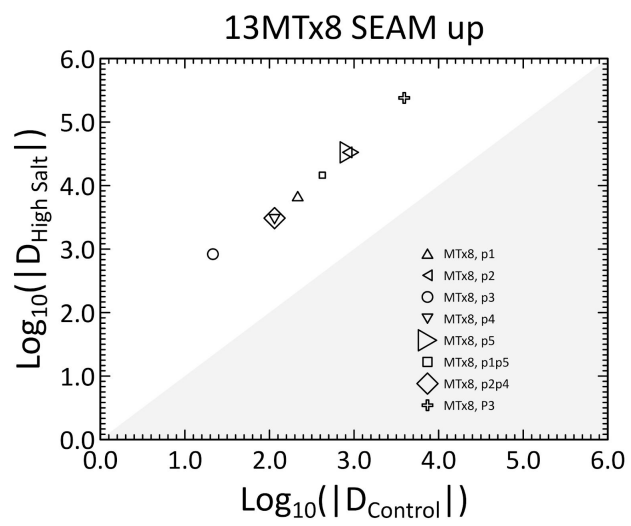

D

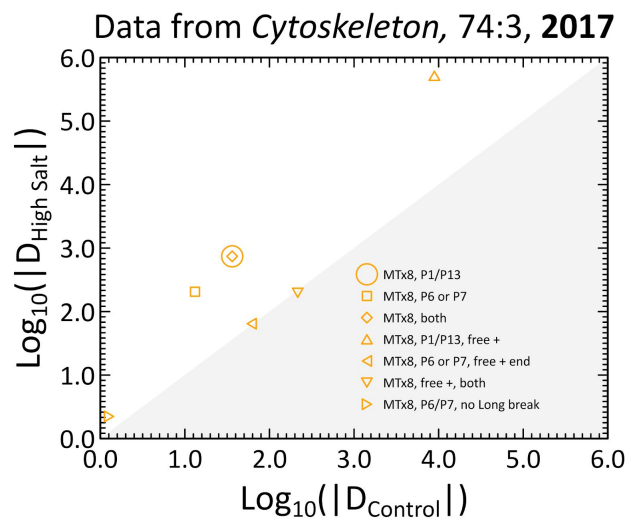

B

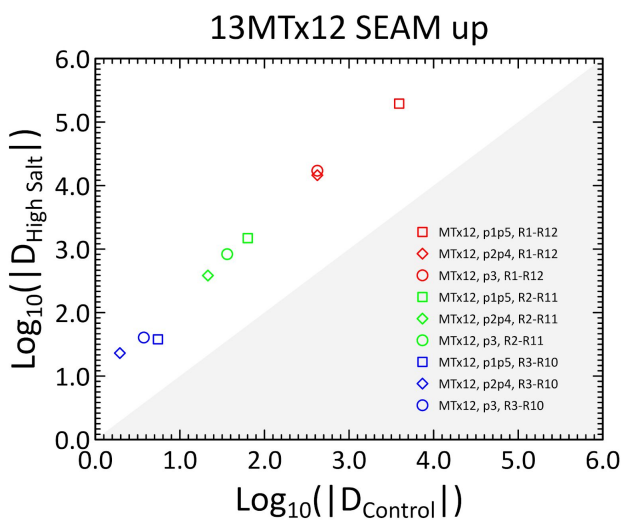

E

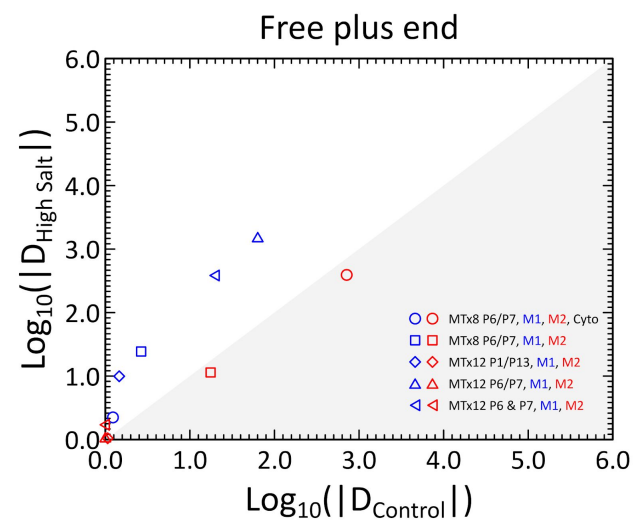

C

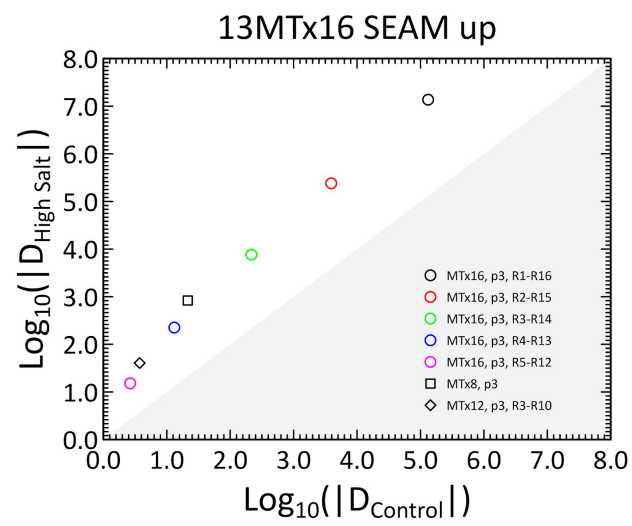

$\mathbf{F}$

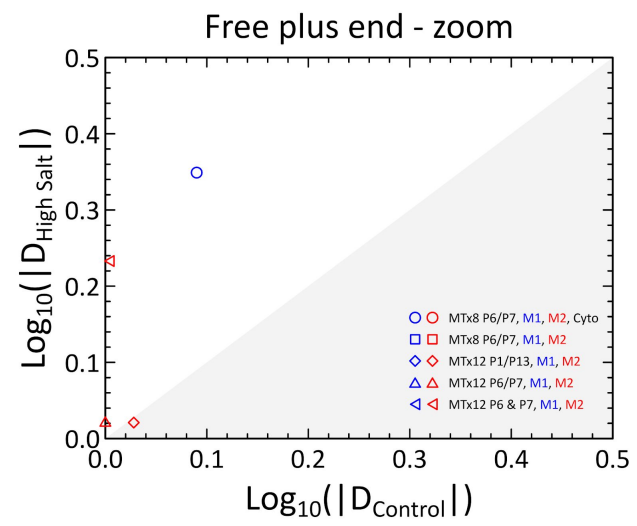

A. KS log-log plots for the 8D seam-up system with shown changes in KS value when we combine position 1 and 5 and positions 2 and 4 together: position 1: pushing between P12/P13; position 2: pusing on P13; position 3: pushing between P1/P13; position 4: pushing on P1; position 5: pushing between P1/P2; P3: pushing on PF P3; B. KS log-log plots for 12D system with shown changes in KS value if the reference dimers were taken from ring 1 and 12 (R1-12) and moved further to the center of the microtubule model (R2-R11 and R3-R10), Description of the position see Fig.S6.A; C. KS log-log plots for 16D system on position 3 (P1/P13) with shown changes in KS value if the reference dimers were taken from ring 1 and $16(\mathrm{R} 1-12)$ and moved further to the center of the microtubule model (R2-R15, R3-R15, R4-R13 and R5-R12). Additional points from the 8D and the 12D MTs are added for comparison. Description of the positions as in Fig.S6.A; D. KS log-log plots for our the KS test analysis of our previous simulations from ${ }^{2}$; E. KS log-log plots for 8D and 12D systems with free plus ends and indentation on different positions (on lateral interfaces between PFs or on a PF) and comparison of two different methods for the collection of angles: M1 - both ends as a reference; M2 - only minus end. MTx8 P6/P7 Cyto - data for simulations in ${ }^{2}$. MTx8 P6/P7 - new runs with a slightly different orientation of the tip; F. Zoom for the KS log-log values from Fig.S6.E.

\section{Fig.S11. Histograms of bending angles}

Histograms of bending angles from simulations (blue) vs. the control (green) and respectively the high salt (orange) data from severing assays. Because of their high similarity, we report positions 1 and 5 , and positions 2 and 4 together. The insets show the corresponding CDFs. 
A. MTx8, both end fixed

position 1 - (P12/P13) and 5 (P1/P2): p1p5; position 2 - (P13) and 4 (P1): p2p4; position 3 - (P1/P13): p3
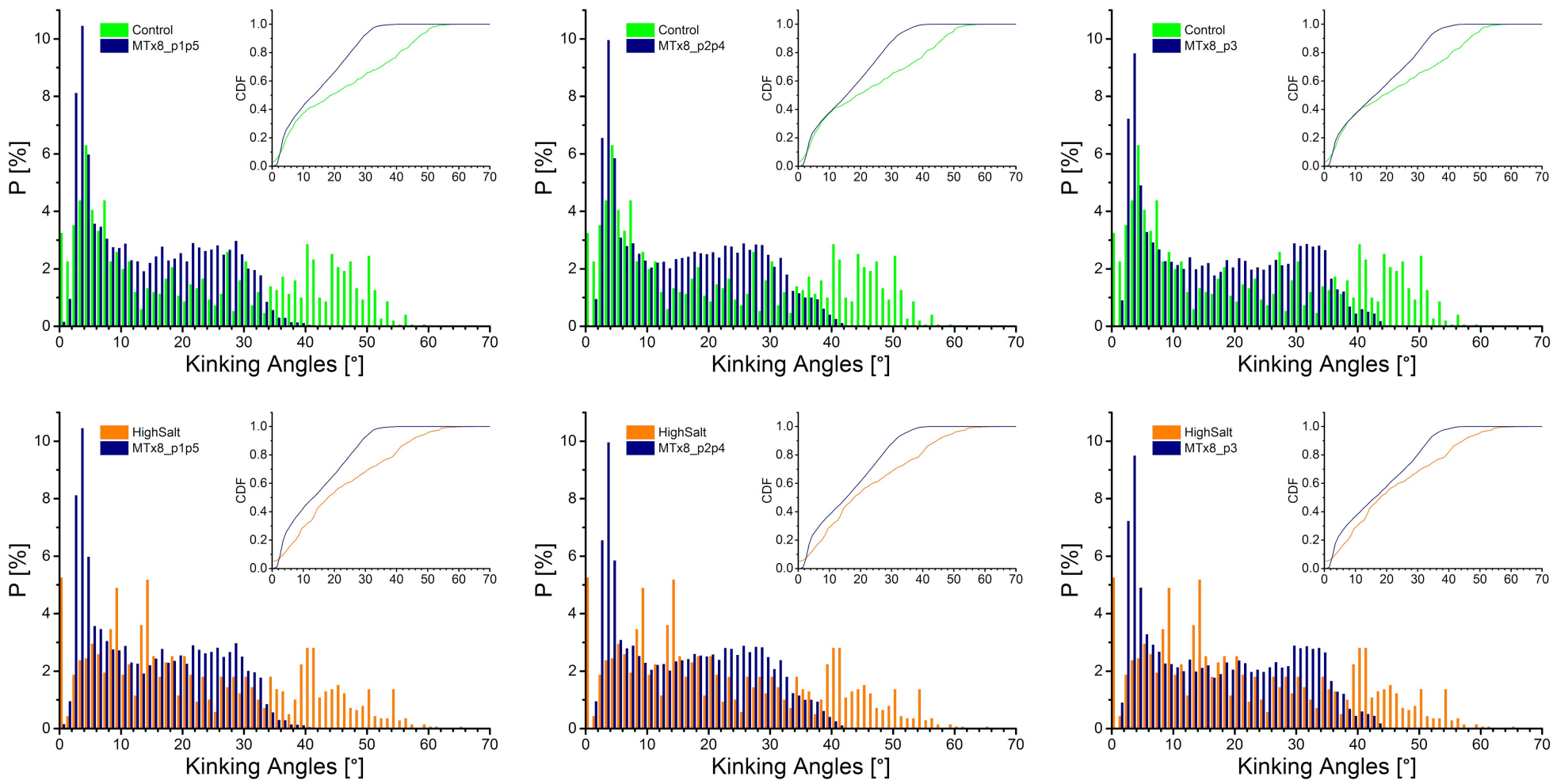

B.. MTx12, both end fixed, R3-R10

position 1 - (P12/P13) and 5 (P1/P2): p1p5; position 2 - (P13) and 4 (P1): p2p4; position 3 - (P1/P13): p3
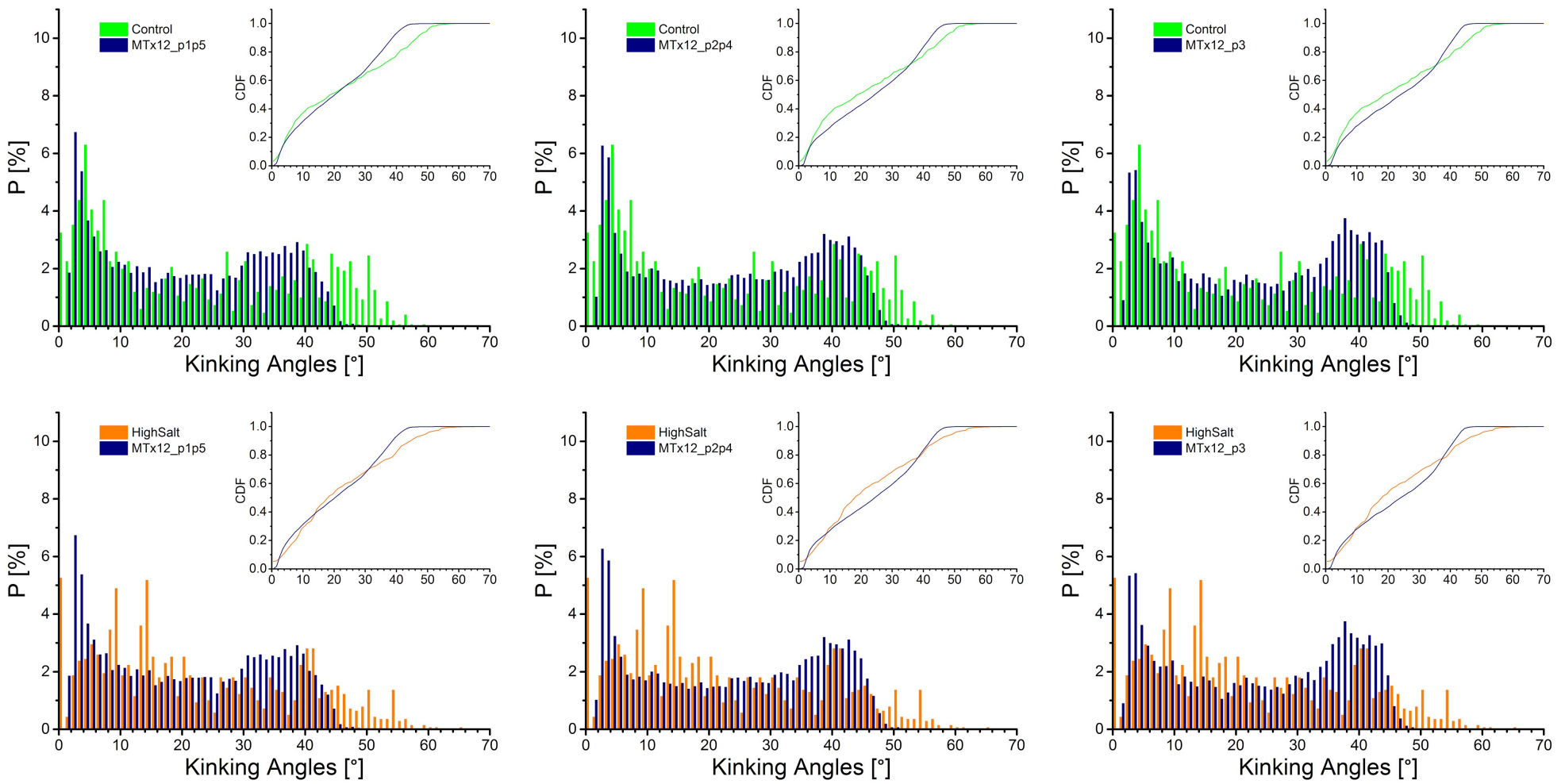
C. MTx16, both end fixed, R5-R12

S- $u$ - position 3 - (P1/P13): p3
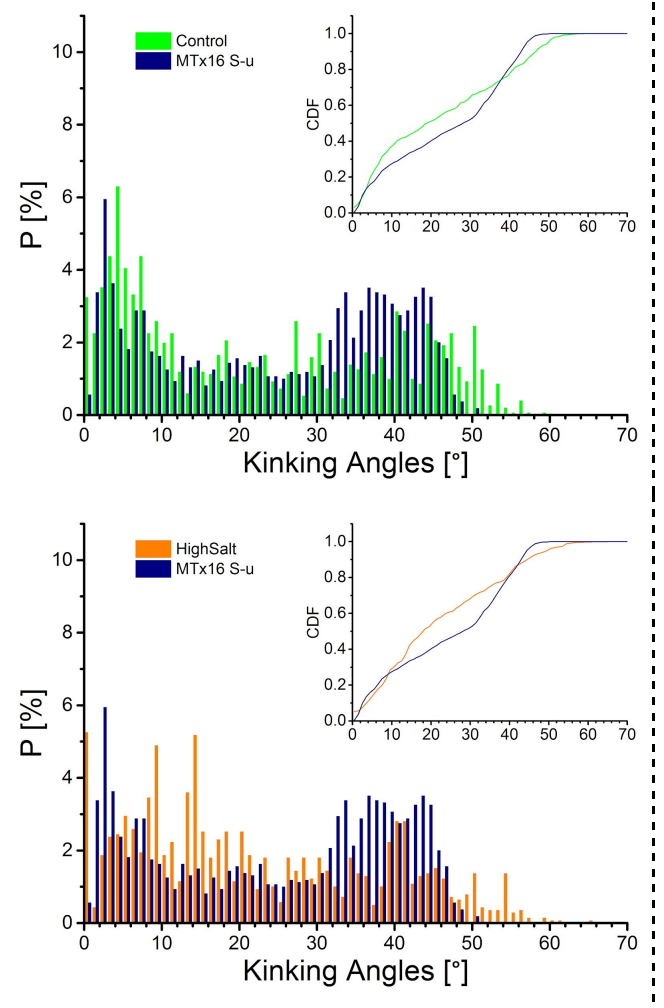

D. MTx8, plus end free;

pusing between protofilaments P6/P7, C - data from Cytoskeleton,
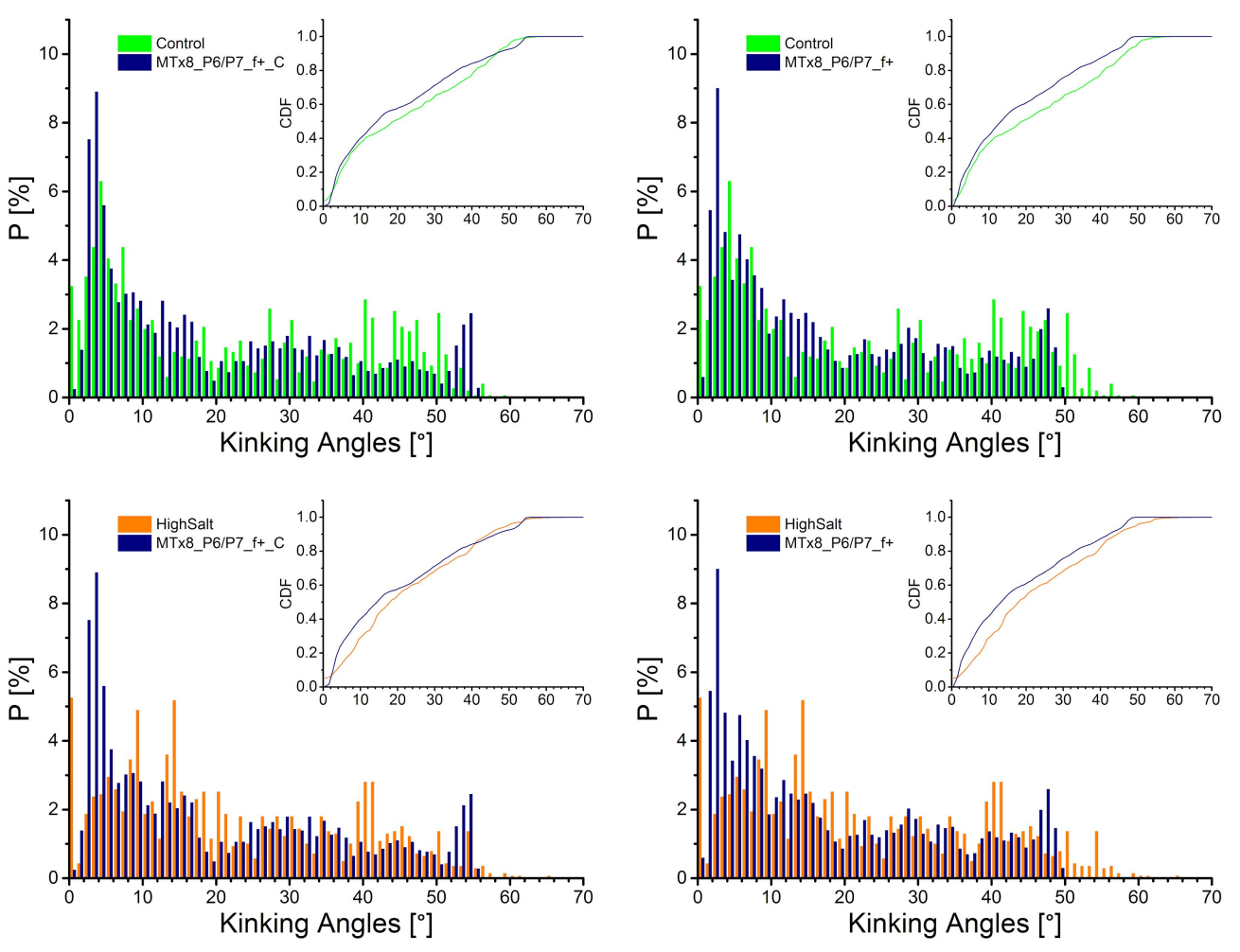

\section{E. MTx12, plus end free;}

S- $u$-> indentation on P1/P13; D71D72 pushing between protofilament P6/P7; D71_\&_D72 -> combined data from pushing on $\mathrm{P} 6$ and $\mathrm{P} 7$
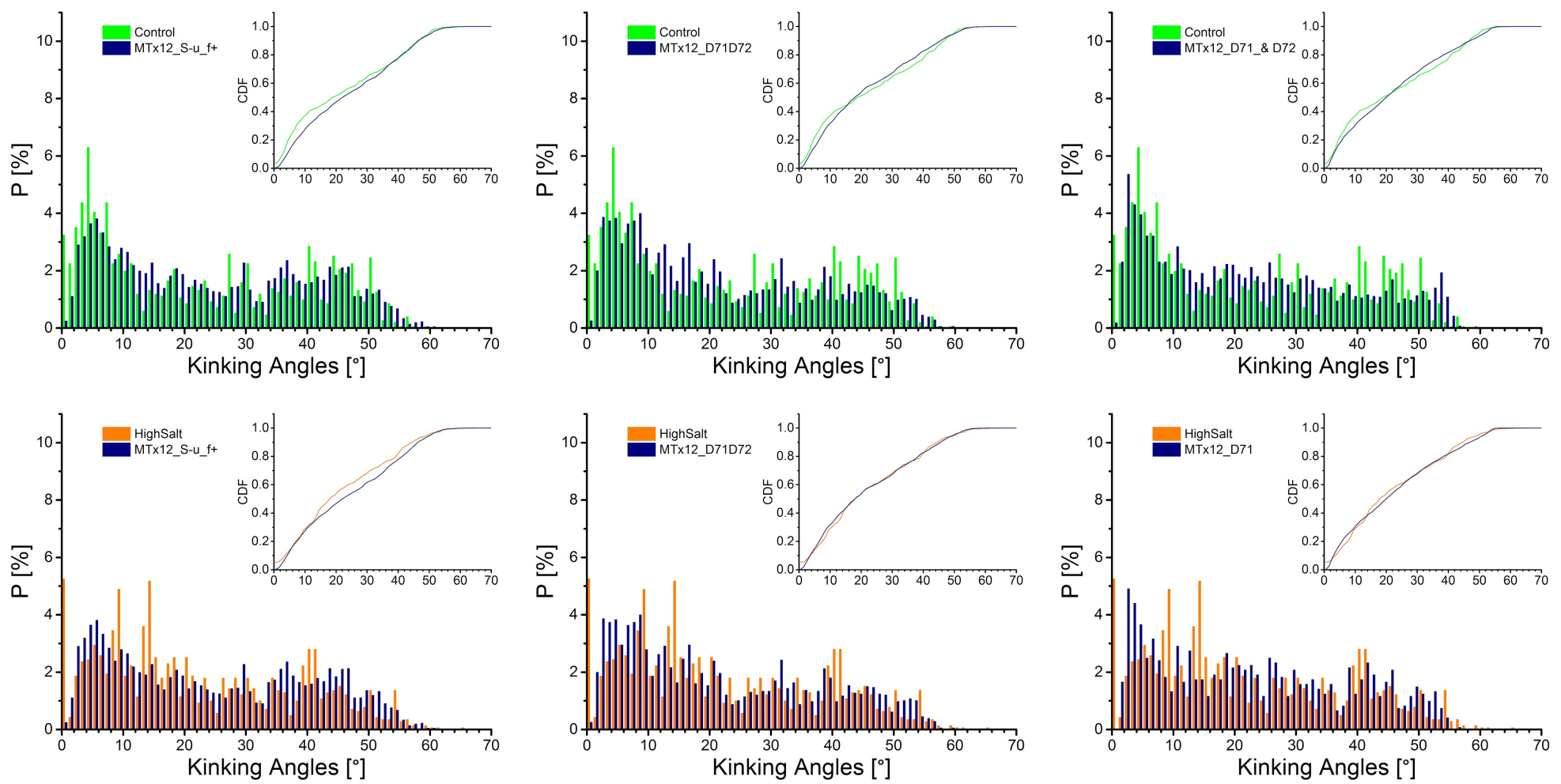
F. MTx8, both end fixed,

$P 3$ - pushing on protofilament P3
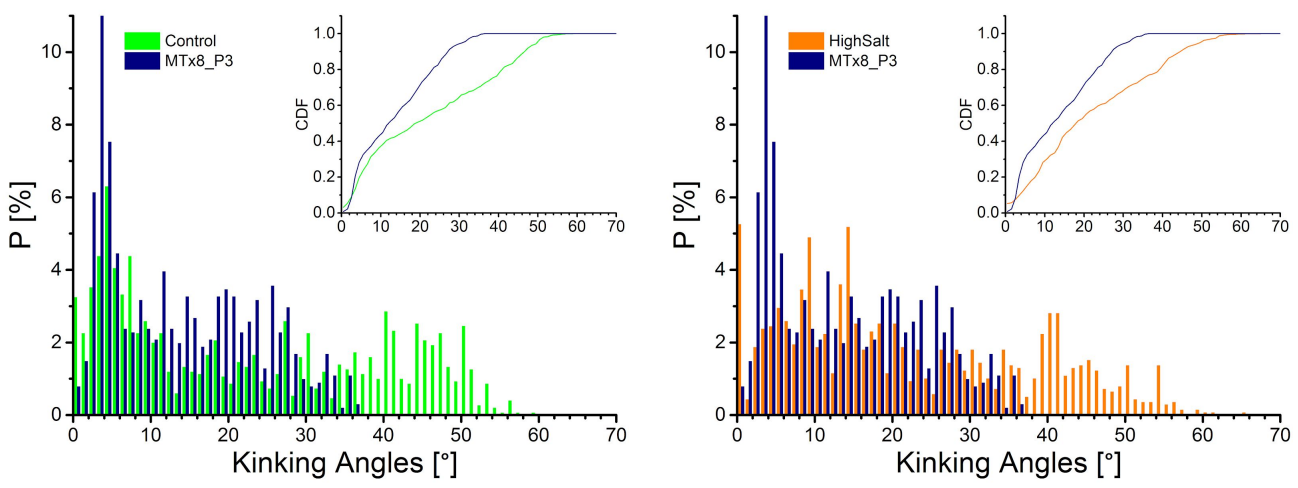

\section{Fig.S12. Retraction and Recovery from Indentation near the seam}
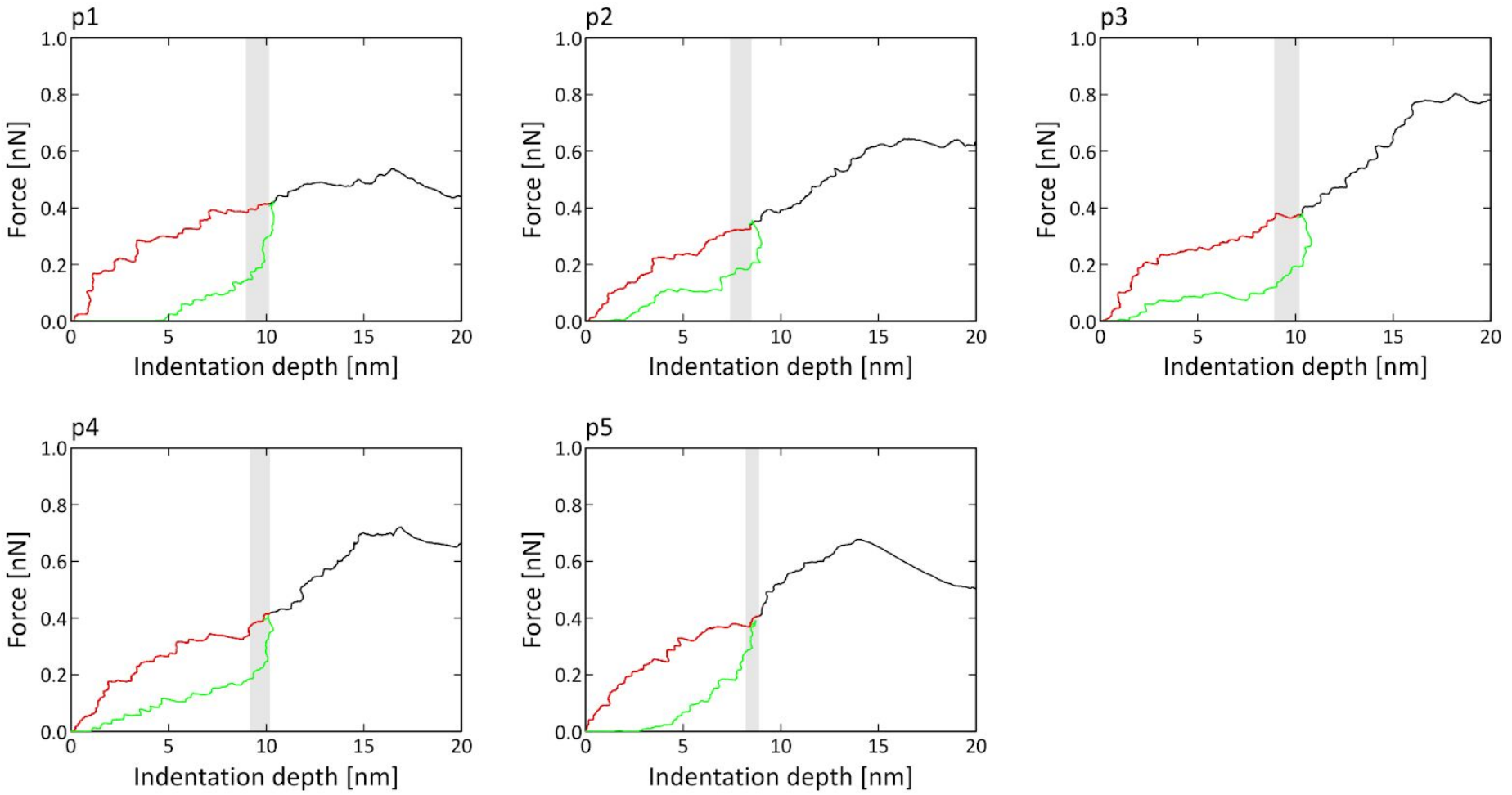

Examples of the force vs. indentation depth plots for indentation-retraction trajectories for all 5 indentation positions near the seam ( $p 1$ to $p 5$ ) for the MT 12D filament. The indentation data is taken from trajectories without the plate. All retraction runs were performed without the plate. Black line - forces for full indentation trajectory; red - forces for the indentation trajectories up to the point where retractions were initialized; grey highlighted region - forces for the indentation trajectories for the indentation depth at which the seam break occurs up to the point where retractions were initialized; yellow - forces for retraction trajectories. 


\section{Fig.S13. Protofilament elongation analysis}

A

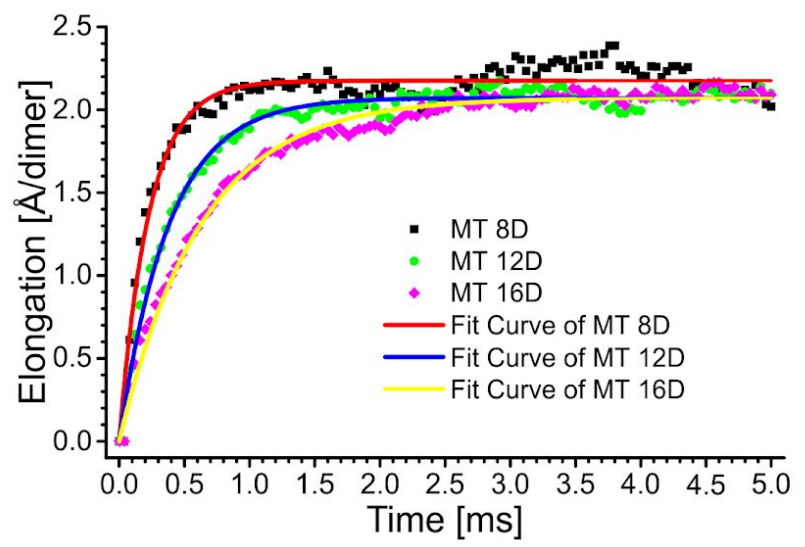

B

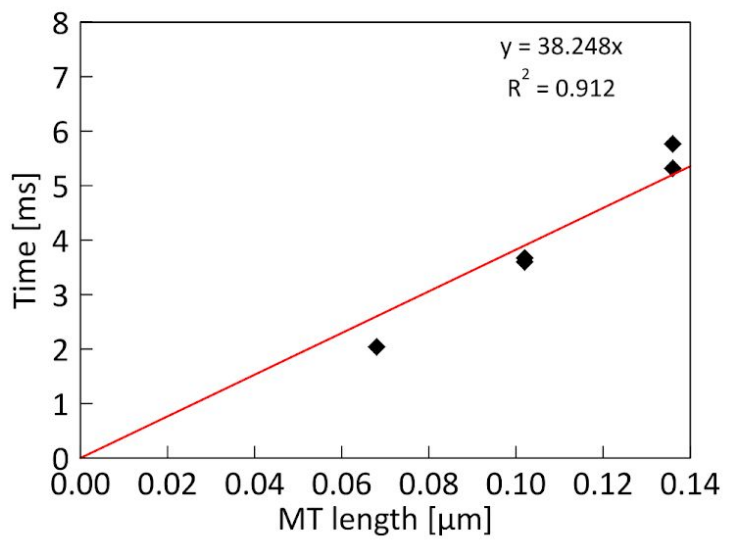

Example plots for Fitting the elongation data using the Levenberg-Marquardt algorithm for 8D, 12D and 16D microtubule polymer without frozen parameter a (panel A) and corresponding fitting of the elongation time vs polymer length for model 3 .

\section{References}

(1) Harris, B. J.; Ross, J. L.; Hawkins, T. L. Microtubule seams are not mechanically weak defects. Phys. Rev. E 2018, 97, 062408-7.

(2) Jiang, N.; Bailey, M.; Burke, J.; Ross, J. L.; Dima, R. I. Modeling the effects of lattice defects on microtubule breaking and healing. Cytoskeleton 2017, 74, 3-17.

(3) Kononova, O.; Kholodov, Y.; Theisen, K. E.; Marx, K. A.; Dima, R. I.; Ataullakhanov, F. I.; Grishchuk, E. L.; Barsegov, $\mathrm{V}$. Tubulin bond energies and microtubule biomechanics determined from nano-indentation in silico. J. Am. Chem. Soc. 2014, 136, 17036-17045.

(4) dePablo, P. J.; Schaap, I. A. T.; MacKintosh, F. C.; Schmidt, C. F. Deformation and collapse of microtubules on the nanometer scale. Phys. Rev. Lett. 2003, 91, 098101-4. 